\title{
WestVirginiaUniversity
}

THE RESEARCH REPOSITORY @ WVU

Graduate Theses, Dissertations, and Problem Reports

2005

\section{Non-ideal iris recognition}

\author{
Vivekanand Dorairaj \\ West Virginia University
}

Follow this and additional works at: https://researchrepository.wvu.edu/etd

\section{Recommended Citation}

Dorairaj, Vivekanand, "Non-ideal iris recognition" (2005). Graduate Theses, Dissertations, and Problem Reports. 1660.

https://researchrepository.wvu.edu/etd/1660

This Thesis is protected by copyright and/or related rights. It has been brought to you by the The Research Repository @WVU with permission from the rights-holder(s). You are free to use this Thesis in any way that is permitted by the copyright and related rights legislation that applies to your use. For other uses you must obtain permission from the rights-holder(s) directly, unless additional rights are indicated by a Creative Commons license in the record and/ or on the work itself. This Thesis has been accepted for inclusion in WVU Graduate Theses, Dissertations, and Problem Reports collection by an authorized administrator of The Research Repository @ WVU. For more information, please contact researchrepository@mail.wvu.edu. 


\title{
Non-Ideal Iris Recognition
}

\section{Vivekanand Dorairaj}

\author{
A Thesis submitted to the \\ College of Engineering and Mineral Resources \\ at West Virginia University \\ in partial fulfillment of the requirements \\ for the degree of \\ Master of Science \\ in \\ Electrical Engineering \\ Natalia A. Schmid, D.Sc., Chair \\ Lawrence A. Hornak, Ph.D. \\ Bojan Cukic, Ph.D. \\ Arun A. Ross, Ph.D. \\ Lane Department of Computer Science \\ and Electrical Engineering \\ Morgantown, West Virginia \\ 2005
}

Keywords: Iris recognition, independent component analysis, principal component analysis, image encoding, performance evaluation, biometrics

(C) Copyright 2005 by Vivekanand Dorairaj

All Rights Reserved 
This page is intended to be blank. 


\section{ABSTRACT}

\section{Non-Ideal Iris Recognition \\ Vivekanand Dorairaj}

Of the many biometrics that exist, iris recognition is finding more attention than any other due to its potential for improved accuracy, permanence, and acceptance. Current iris recognition systems operate on frontal view images of good quality. Due to the small area of the iris, user co-operation is required. In this work, a new system capable of processing iris images which are not necessarily in frontal view is described. This overcomes one of the major hurdles with current iris recognition systems and enhances user convenience and accuracy. The proposed system is designed to operate in two steps: (i) preprocessing and estimation of the gaze direction and (ii) processing and encoding of the rotated iris image. Two objective functions are used to estimate the gaze direction. Later, the off-angle iris image undergoes geometric transformations involving the estimated angle and is further processed as if it were a frontal view image. Two methods: (i) PCA and (ii) ICA are used for encoding. Three different datasets are used to quantify performance of the proposed non-ideal recognition system. 
This page is intended to be blank. 
To my family. 
This page is intended to be blank. 


\section{Acknowledgements}

This thesis is a result of work that was done in the past two years, starting in the second semester of my Master's program. I received help from many quarters in order to successfully complete this task and I would like to thank all those who had helped me with this thesis work from the beginning.

Firstly, I take pleasure in expressing my heartfelt gratitude to my adviser $D r$. Natalia Schmid. She has been highly inspiring, soft spoken yet strict friend who was always there when I needed her help. She has been extremely patient with me and has guided this work. I do not know how well this work would have gone if not for her help and encouragement. It has been a great previlege to be her student.

I would like to thank all the committee members, namely Dr. Larry Hornak, Dr. Bojan Cukic and Dr. Arun Ross for their continued support throughout my study. I am indebted to all the three for their constant encouragement and wise suggestions which helped carve not only my thesis but my approach towards quality research. I have learnt a lot from my committee members, sometimes even without their knowledge.

I extend my special gratitude to all my family members, Appa, Amma, Prakash, Sangeetha, Arun, and Ram for standing by me during this journey that I undertook, for all their love and care. Without their unwitting support, this thesis wouldn't have been possible.

Special thanks to all my friends and colleagues at work, especially Nathan, Jinyu, Xiaohan and Nick. It has been a great experience to share knowledge and work as a team with all of you.

I would like to take this opportunity to thank Prof. John Daugman for his valuable 
suggestions which added greatly to my understanding of iris recognition.

Last but not the least, I would like to thank the CITeR and the Biometric Fusion Center for sponsoring this project. 


\section{Contents}

Acknowledgements vii

1 Introduction 1

1.1 Biometrics ........................... 1

1.1.1 Physiological Biometric . . . . . . . . . . . 2

1.1.2 Behavioral Biometric ................ 2

1.1.3 Templates ..................... 3

1.1.4 Modes of Operation . . . . . . . . . . . . . 3

1.1 .5 Iris Recognition . . . . . . . . . . . . . . . . . . 4

2 Literature Review $\quad 6$

2.1 Localization . . . . . . . . . . . . . . . . . . . 8

2.2 Normalization . . . . . . . . . . . . . . . . . 8

2.3 Encoding ............................ 9

2.4 Matching ...................... 10

3 Non-Ideality in Iris Recognition $\quad 11$

3.1 Estimation of Projective Transformation Matrix . . . . . . . . . . 13

3.2 Ellipse Fitting for Angle Estimation . . . . . . . . . . . . . . . . 15

4 Proposed Encoding Techniques 17

4.1 Encoding Using PCA . . . . . . . . . . . . . . . . . . 17 
4.2 Following up with ICA . . . . . . . . . . . . . . . 22

5 Results $\quad 25$

5.1 Performance of Estimation techniques . . . . . . . . . . . . 25

5.2 Results for Encoding of Frontal View Images . . . . . . . . . . . . . . 30

5.2.1 Compensation for Rotation . . . . . . . . . . . 30

5.2.2 Occlusion, Masking and Performance . . . . . . . . . . 32

5.3 Performance of the Non-ideal Recognition system . . . . . . . . . . 35

5.4 Other Studies on Encoding . . . . . . . . . . . . . . . . . . . 38

5.4.1 How many Eigenvectors? . . . . . . . . . . . . . . . . . 40

5.4 .2 Eigen irises and Independent irises . . . . . . . . . . . . . . 40

5.4.3 Effect of Varying the Number of Essential Components . . . . 42

5.4 .4 Identification case with top $\mathrm{N}$ matches . . . . . . . . . . . 43

5.4.5 Relative Composition of Training and Testing Sets . . . . . . 44

5.4.6 Effect of Varying the Resolution . . . . . . . . . . . . . . . 45

5.4.7 Other Databases and Distance Measures . . . . . . . . . . . 47

5.5 Effect of Specular Reflection on Performance . . . . . . . . . . . 47

6 Summary and Future Work $\quad 49$

6.1 Summary .............................. 49

6.2 Future Work . . . . . . . . . . . . . . . . 50

$\begin{array}{ll}\text { Bibliography } & 51\end{array}$ 


\section{List of Figures}

1.1 Currently used biometric technologies . . . . . . . . . . . . . . . 2

1.2 Anatomy of the iris (from http://www.lab.anhb.uwa.edu.au/vsci/IRISCB.htm) 5

2.1 Examples of iris images from two different databases. On top, we have three samples from CASIA dataset and at the bottom, we have three samples from a dataset collected in our labs at WVU. . . . . . . . . . 7

2.2 Schematic of the modified Daugman's algorithm . . . . . . . . . 8

3.1 Examples of non-ideal iris images . . . . . . . . . . . . . . . 12

3.2 A generic Non-Ideal Iris based Recognition System . . . . . . . . . 13

3.3 A Schematic of a Non-Ideal Iris based Recognition System which deals with off-axis images . . . . . . . . . . . . . . . . . . . 14

4.1 Block-diagram of the system implementing PCA/ICA encoding techniques for iris. . . . . . . . . . . . . . . . . . . . 18

4.2 Schematic of testing scenario 1 . . . . . . . . . . . . . . . . . 19

4.3 Schematic of testing scenario 2 . . . . . . . . . . . . . . 21

5.1 Estimating Off-Angle Using Projective Transform with one axis of rotation using Hamming Distance as criterion . . . . . . . . . . . 26

5.2 Estimating Off-Angle Using Projective Transform with one axis of rotation using Integro-differential operator . . . . . . . . . . .

5.3 Estimating Off-Angle Using Projective Transform with two axes of rotation . . . . . . . . . . . . . . . . . . . . . 28 
5.4 Estimating ellipse parameters using the elliptical integro-differential operator . . . . . . . . . . . . . . . . . . . 29

5.5 Performance in different scenarios . . . . . . . . . . . . . . . . . . 32

5.6 Effect of rotation on matchscores, (a).Histogram before compensation, (b).Histograms after compensation . . . . . . . . . . . . . 33

5.7 PCA/ICA results for various simulations, (a).ROC curves for PCA, (b).ROC curves for ICA . . . . . . . . . . . . . . . . . . . . 33

5.8 Some examples of masked images . . . . . . . . . . . . . . 35

5.9 Some examples of Independent irises . . . . . . . . . . . . . . . 36

5.10 Some examples of Eigen irises . . . . . . . . . . . . . . . 36

5.11 Effect of applying a unified mask to reduce interference from eyelids . 36

5.12 Percentage variance contributed by each eigen vector to the total variance as a function of (a) Varying resolution of training image with constant number of training templates (b) Varying number of templates for constant resolution . . . . . . . . . . . . . . . . .

5.13 Three samples of a single user from an off-axis dataset collected in our

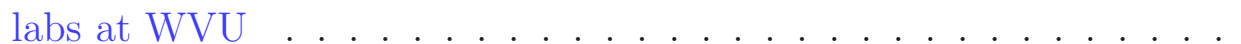

5.14 Performance of algorithms when using initial estimates for angles. (a). Train on 0 degree images and tested on others, and (b). Train on 15 degree images and tested on others . . . . . . . . . . . . 38

5.15 Performance of algorithms after compensating using the optimization technique (integro-differential operator). (a). Train on 0 degree images and tested on others (b). Train on 15 degree images and tested on others, and (c). Train on 30 degree images and tested on others . . .

5.16 Plot of image after projection into different trained subspaces . . . . 41

5.17 Verification performance with varying number of essential components and number of users . . . . . . . . . . . . . . . . . . . . . 42

5.18 Identification performance with varying number of essential components and number of users . . . . . . . . . . . . . . . . . 42 
5.19 (a). Recognition performance with top $\mathrm{N}$ matches. M- with masking for occlusions, NM-without masking for occlusions. (b).Performance of proposed techniques as the number of training images in identification case. There is clearly an improvement in performance when using more training templates. . . . . . . . . . . . . . . . 43

5.20 Downsampling of a normalized iris image . . . . . . . . . . 45

$5.21 \mathrm{PCA} / \mathrm{ICA}$ results for various simulations on resolution $\ldots \ldots . . .46$

5.22 Verification performance for WVU dataset with 22 iris classes and six images per user . . . . . . . . . . . . . . . . 47

5.23 Effect of presence of specular reflection on performance of algorithms 48 
This page is intended to be blank. 


\section{Chapter 1}

\section{Introduction}

Authentication can be formally defined as the process of determining whether or not a given person is indeed who he/she claims to be. We need authentication in an environment where only the right person has access to a given resource. In order to determine the identity, the knowledge of some undisclosed password or the possession of some documents or cards is commonly used. But passwords can be guessed or retrieved, documents forged and cards stolen. Hence, we need a reliable way to differentiate between individuals as well as identify them based on information unique to each person. One such highly unique feature which provides a reliable way of determining who an individual is rather than what the individual knows or has, is the biometric trait of a person.

\section{$1.1 \quad$ Biometrics}

Biometrics are automated methods of verifying or identifying an individual based on physiological or behavioral characteristics of that person. Currently, there are a number of biometrics which can be used in three modes, (1) verification, (2) identification, and (3) screening. Biometrics are classified into two types based on the type of input: (1) physiological, and (2) behavioral. 

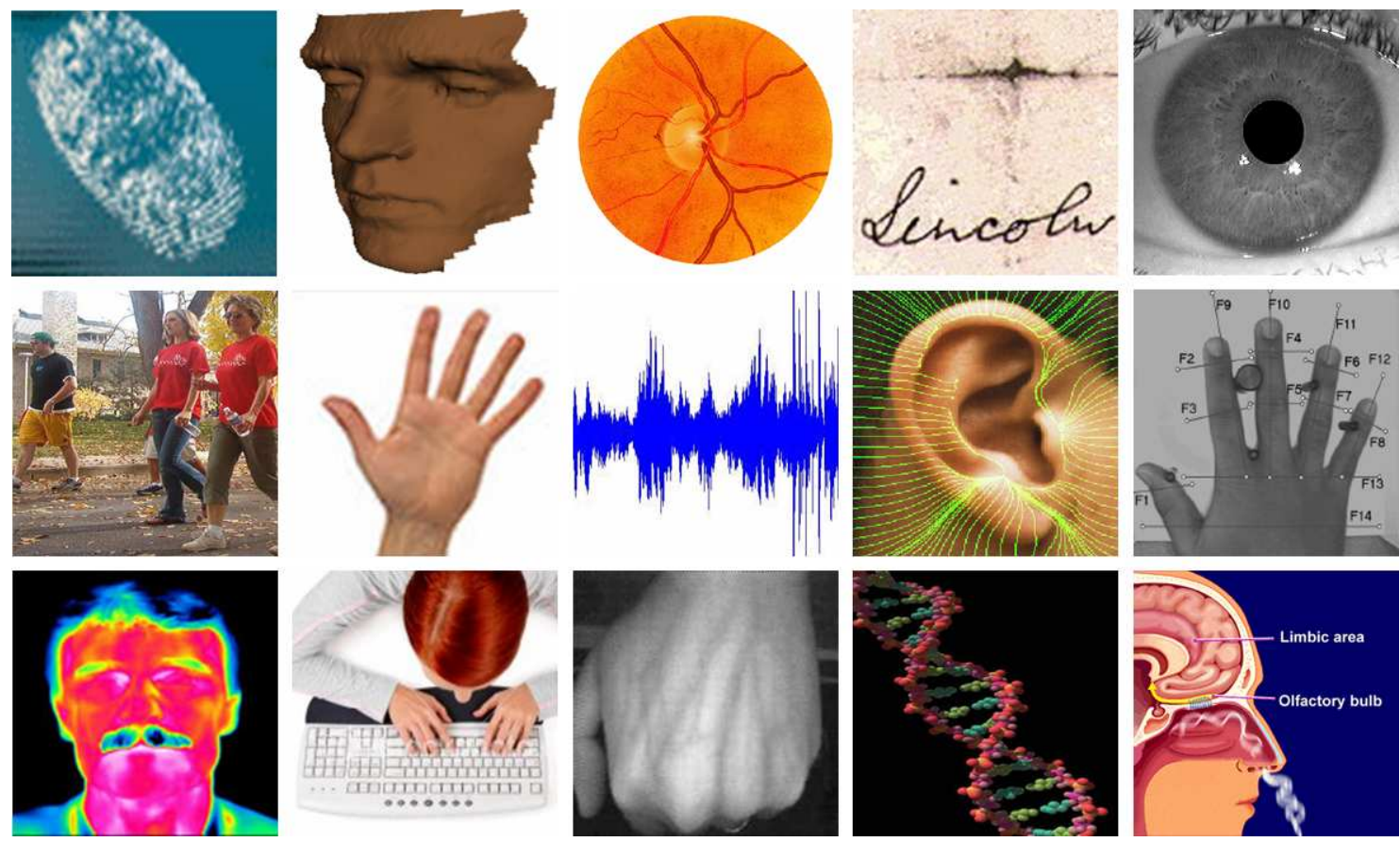

Figure 1.1: Currently used biometric technologies

\subsubsection{Physiological Biometric}

If the biometric recognition relies on variability in physical appearance of certain visible body parts, then it is called a physiological biometric. Features used for recognition are usually obtained from images either in the visible or infrared spectrum. Examples of these include fingerprint, iris, hand geometry, face, palmprints, retina, dental etc. These biometric traits usually give better performance than behavioral biometric though its usage in a given situation may depend on what the need is. These are usually non-replacable and hence any raw data or template associated with this type of biometric requires additional security measures, for instance using watermarking or cryptographic techniques.

\subsubsection{Behavioral Biometric}

Any action of a person is called a behaviour and is generally different from how a person looks like though appearance may be an influencing factor in one's behaviour. 
Certain kinds of behaviour such as signature, gait, etc. are considered unique among individuals. Hence, these traits are used for biometric recognition. Currently, the performance of such recognition systems are poor than physiological biometric but their importance cannot be ignored for certain applications. For instance, monitoring a large group of persons at a distance for "unusual" behaviour is a typical application for gait recognition. This may not be possible for other forms of biometrics like face (can be easily altered), fingerprints, ear, and iris recognition (no input of biometric) etc. They can also aid in multimodal biometric systems.

\subsubsection{Templates}

Templates form the heart of any biometric system. A template is a set of features often in the form of a vector, which is extracted from the original input to the biometric system. Some examples of templates are cepstrum coefficients from voice data, fingerprint minutiae, eigen coefficients for face, etc. There are several advantages of extracting such features from a given biometric. It generally reduces storage space though this may not be possible in certain cases. For example, the use of overcomplete representations such as steerable pyramids. Templates usually make the original raw data better suited for classification. Templates in turn form the input for the classification step. In general, features extracted using sophisticated techniques may require only simple classifiers.

\subsubsection{Modes of Operation}

There are three main modes in which a recognition task is performed. They are:

Verification: Given an input of some kind (may be password or a biometric), the task is to verify whether or not a person is really whom he/she claims to be. This is a one-to-one match and the final output is a binary decision.

Identification: Given an input which is thought to be unique for individuals, the task is to identify the person to whom the given input belongs to provided that 
another copy of the input is available in the database. This is a one-to-many match with either one or more closest matches as the output.

Screening: Screening is defined as testing objects or persons in order to identify those with particular characteristics. In this case, the task is to say whether or not the given input belongs to one of the individuals present in a watch list. Here the number of comparisons is greater than that of the verification case but fewer than the identification case.

\subsubsection{Iris Recognition}

A highly complex phenomenon due to the combined effects of texture, pigmentation, fibrous tissue, and blood vessels within the iris stroma make up an individual's epigenetic constitution. Iris pattern, unlike biometrics such as fingerprints or face, does not contain predefined sets of features. For example, a face has two eyes, one nose, a mouth and two ears with a fixed "relative order". Fingerprint consists of ridges and valleys with a limited set of minutiae patterns (such as ridge ending, ridge bifurcation etc.) positioned randomly (with varying numbers) for different individuals. Iris on the other hand does not have such restrictions. It combines numerous local pattern variations (varying in scale and position) along with global features to give a highly unique pattern. Recognition systems based on such patterns have been proven to have greater accuracy than many of the existing biometrics [1]. They were also observed to be stable over a considerable period of a person's lifetime.

\section{Iris Anatomy}

The human eye is a complete imaging system and the iris is an integral part of it. The iris is a contractile diaphragm that controls the degree of retinal illumination and consists of the following layers from anterior to posterior:

Anterior border layer: This layer is loose, thin and avascular. It contains densely packed pigmented and nonpigmented cells not covered by true epithelium but discontinuous layer of fibroblasts. 


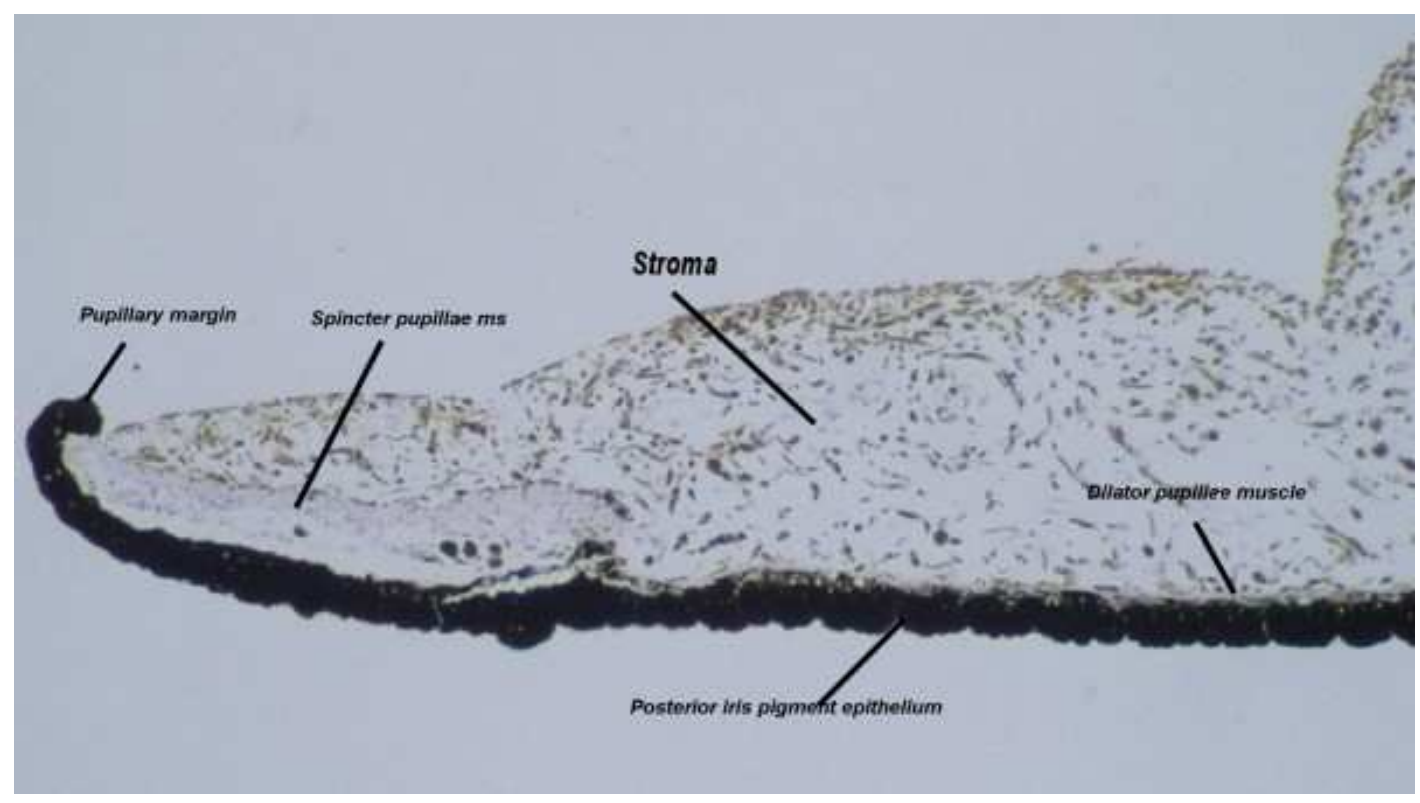

Figure 1.2: Anatomy of the iris (from http://www.lab.anhb.uwa.edu.au/vsci/IRISCB.htm)

Stroma: This layer is thicker and highly vascular. It contains delicate collagenous fibers intermixed with varying proportions of pigmented and nonpigmented cells.

Sphincter pupillae muscle: This is a smooth, concentrically arranged muscle responsible for constriction of pupil by contraction.

Myopigmented anterior epithelial layer: This is the same layer as the outer pigmented epithelium of ciliary body and consists of peculiar pigmented cuboidal cells. These cells and their processes form the Dilator pupillae muscle (thin and inconspicuous), which performs the dilation of the pupil in dark environments.

Posterior epithelial layer: This layer extends the inner epithelial nonpigmented cell layer of ciliary body. Here the cells are heavily pigmented and simple columnar cells are loosely attached to anterior epithelial layer. 


\section{Chapter 2}

\section{Literature Review}

The first iris recognition system was invented by John Daugman $[2,3,4,5]$ as an application to his findings and theory on two dimensional visual cortical filters. The systems described in most of the iris recognition literature captures and further processes either single or multiple copies of infra-red or visible light frontal view images of high resolution with no strong blur or occlusions in order to guarantee good performance. Most of the systems are patented. There are no details provided on how to select certain parameters for implementing Daugman's system. The literature contains a large number of publications that try to explain the different parts of $\mathrm{J}$. Daugman's system $[2,6,7,8]$. The following general steps are involved:

1. Localization of region of interest: During this step, the pupil, sclera, and eyelids are segmented.

2. Normalization: Transforms a localized iris region from Cartesian coordinates to doubly dimensionless polar coordinates.

3. Encoding: Uses 2D Gabor wavelets to encode image content that is then quantized to two levels based on phase information of the output. The result of encoding step is presented as a binary template called "Iris Code".

4. Matching: Matching is performed using the Hamming distance. 

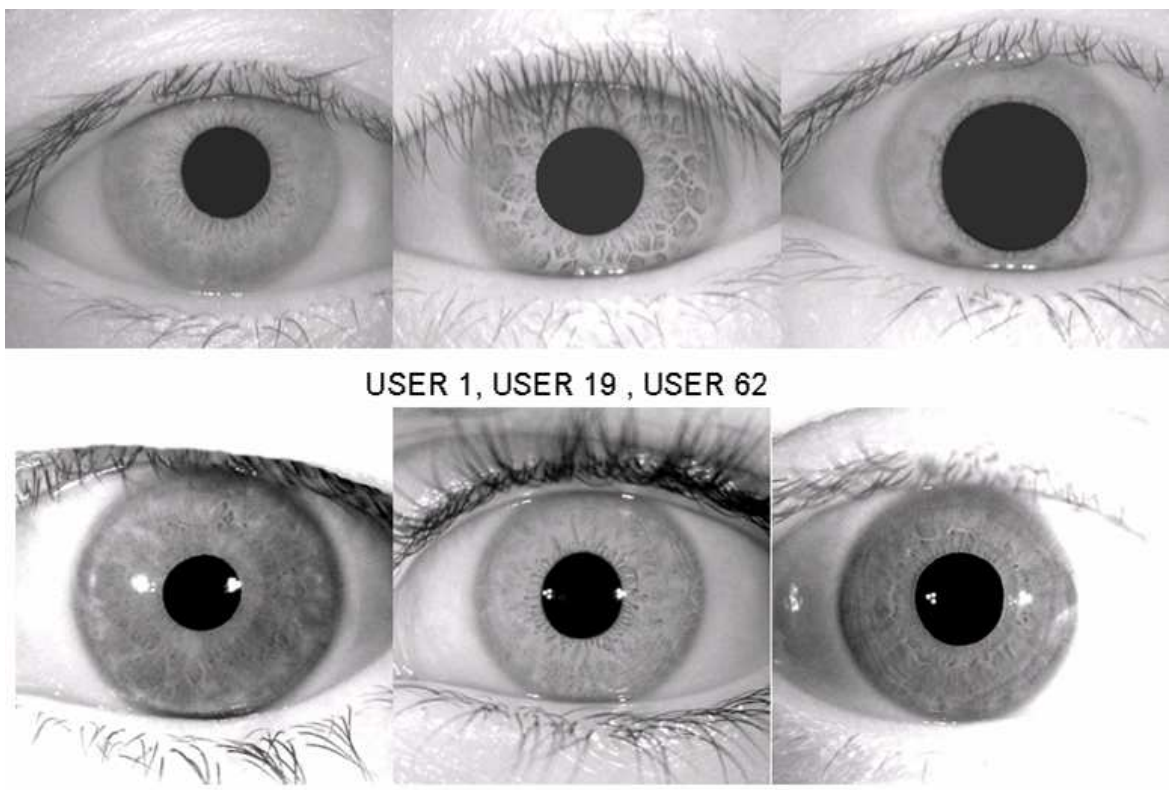

USER 2, USER 3 , USER 7

Figure 2.1: Examples of iris images from two different databases. On top, we have three samples from CASIA dataset and at the bottom, we have three samples from a dataset collected in our labs at WVU. 


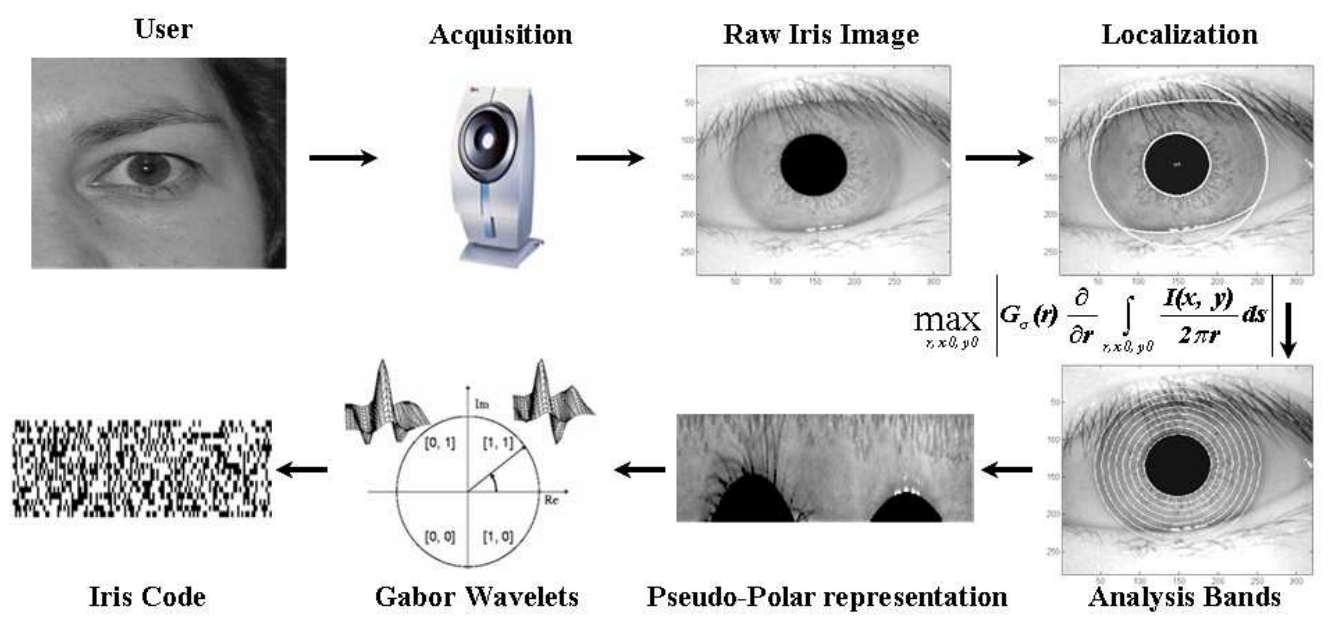

Figure 2.2: Schematic of the modified Daugman's algorithm

A modified version of such a system was implemented at WVU and the results from this implementation serve as a baseline for comparison. A schematic of the system is shown in Fig.2.2.

\subsection{Localization}

Localization is the process of segmenting the iris region from a background of pupil, eyelids, eyelashes etc. Daugman employed an integro-differential operator for performing this step [2]. Other techniques involved for a similar purpose are Hough transform, Haar wavelets, Canny edge detector, active contours etc [7, 9, 10, 11]. Though most of the techniques work well, their performance in most cases can be improved based on prior knowledge about the sensor and by the use of heuristics.

\subsection{Normalization}

Normalization is the process of generating a representation of the localized iris image that is invariant to scale and translation. It can also be made invariant to rotation and other transformations (both linear and non-linear). This representation can be 
achieved by a transformation of the localized image with different techniques. Daugman [2] employs a doubly dimensionless polar representation by the use of a rubber sheet model. Wildes [7], uses a minimization technique based on least squares to obtain image registration. Boles $[12,13]$ relies on the maximum possible outer radius for a given pupil radius to perform normalization. Both Wildes and Boles' technique do not compensate for pupil dilation. Also, Boles' method does not carry out a detection procedure for the outer boundary. Though Wildes' technique does not compensate for pupil dilation, it minimizes loss of information content due to sampling, similar to Daugman's system.

\subsection{Encoding}

Encoding is employed after two important preprocessing steps, namely localization and normalization of the iris region in an image. Daugman derived a general uncertainty principle for 2D filters and proved that the family of 2D Gabor functions achieves the lower bound on joint 2D resolution $[14,15]$. This led to the representation of images using such 2D Gabor elementary functions. Gabor basis functions are not orthogonal, but their coefficients can be calculated easily as described in [2], and can be extended to a wavelet like expansion. This could also be viewed as a demodulation technique which lowers the complexity of the image or as an entropy reducing mechanism to obtain a compact yet efficient representation. Daugman performs recognition based on the failure of test of statistical independence after localization, normalization, and encoding of the iris pattern of the human eye. This is performed not on the original Gabor coefficients, but on the phase quantized output coefficients. Rotation due to head tilt is compensated during matching stage by rotation of the obtained iris template.

Encoding techniques developed by Wildes [7] and Boles [12, 13] use Gaussian pyramids and zero-crossings of wavelets, respectively. There have been a number of techniques for iris recognition in the recent years [16, 17, 18, 19, 20, 21, 22, 23, 24, 25, $26,27,28]$. It is beyond the scope of this work to explain in detail the methodologies presented in these works. The reader is strongly encouraged to refer to at least a 
few of these works to have a better understanding of iris recognition techniques in the literature. There have been ICA based iris recognition techniques in the past. The work presented here is different from both these previous works. Like other previous techniques, the ICA techniques proposed in [29, 30, 31] also encode local information, whereas this work tries to perform global encoding by considering the entire (segmented) iris image as one signal.

In [29], randomly selected local 2D patches (square) are used for training. In $[30,31]$, randomly selected local 2D patches (rectangular) are averaged in the radial direction to obtain 1D signals which are used for training 1D ICA basis functions. From the trained basis, both the above mentioned methods encode the testing images and perform matching. Both these techniques cannot compensate for rotations (inplane and out-of-plane) though they compensate for occlusion. In contrast the method proposed in this work does not require random sampling but rather considers each image as a data point in a high dimensional space.

\subsection{Matching}

Daugman uses Hamming distance between two given templates as the decision criterion. Wildes uses normalized correlation coefficient as the criterion whereas Boles uses a predefined dissimilarity measure at different scales to give a final score. The matching criterion varies according to the feature set. Hamming distance remains the easiest and fastest to calculate across a huge number of templates. 


\section{Chapter 3}

\section{Non-Ideality in Iris Recognition}

The term non-ideal iris recognition refers to an iris recognition system which can effectively deal with iris images of degraded quality due to:

1. User's disability and/or non-compliant behaviour,

2. Low cost acquisition device.

Current iris recognitions systems that have been previously described require good quality frontal view images. This is one reason why iris recognition systems have a high Failure To Acquire (FTA) rates as well as less user convenience. Though there are some systems that avoid the need for users to present their iris very close to the acquisition device, the user still needs to co-operate and present his/her iris to the device such that the system is able to acquire a good frontal view image. This is achieved by assuming users' compliance and the use of good quality sensors. When these conditions are not met, it results in images which contain one or more distortions affecting the performance of the system. In Fig.3.1, some iris images of low quality are shown. These images have (a) bad lighting, (b) distortion due to blurring, (c) outof-plane rotation, respectively. When encountering such inputs, the performance of traditional systems which require good quality input suffers. Hence, there is a need to develop algorithms that can identify individuals based on iris images, irrespective of minor variations in image quality if not major degradation. But there are no publicly available iris image databases which could serve to provide data for studying all such 
factors which affect the performance. The impact of non-ideal imagery is the focus of some current works $[32,33,34,35]$. Of special interest are iris images which are not in frontal view. To effectively deal with any such non-ideal conditions jointly would be the ultimate goal of modern iris recognition systems.
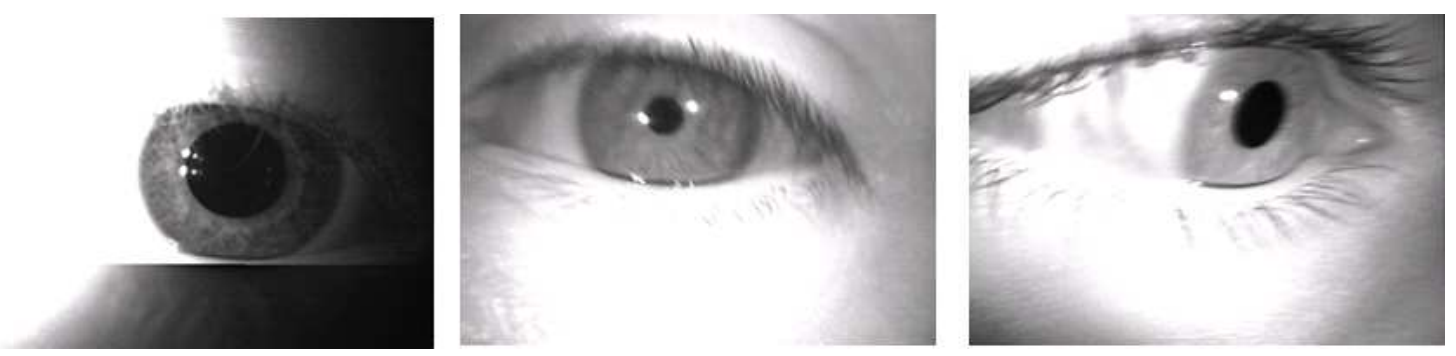

Figure 3.1: Examples of non-ideal iris images

The problem of fitting models to images using image features has been studied previously by other researchers [36, 37, 38, 39, 40]. But they either deal with a set of feature points to which a transformation needs to be estimated or have previously available 3D model for which they know the true parameters. Using this information, the projections of the 3D models (commonly belonging to rigid objects such as man made tools) are compared with the information available from the $2 \mathrm{D}$ image to accomplish the task. In this work, a projective transformation approach is taken in order to deal with off-axis iris images but using information on geometric properties of the human eye. The difference is that the model is not rigid. A similar approach to detecting the human eye using deformable templates has been carried out in [41] but only for frontal view iris images. The model is a 2D model. Moreover, the number of model parameters are significantly larger. The projective transformation can be expressed in terms of the angles of rotation alone if translation and in-plane rotation are not considered. Note that this only means that the translation parameters are implicitly calculated when optimizing for the objective function to locate the iris after rotation is performed. The rotation matrix is given by 


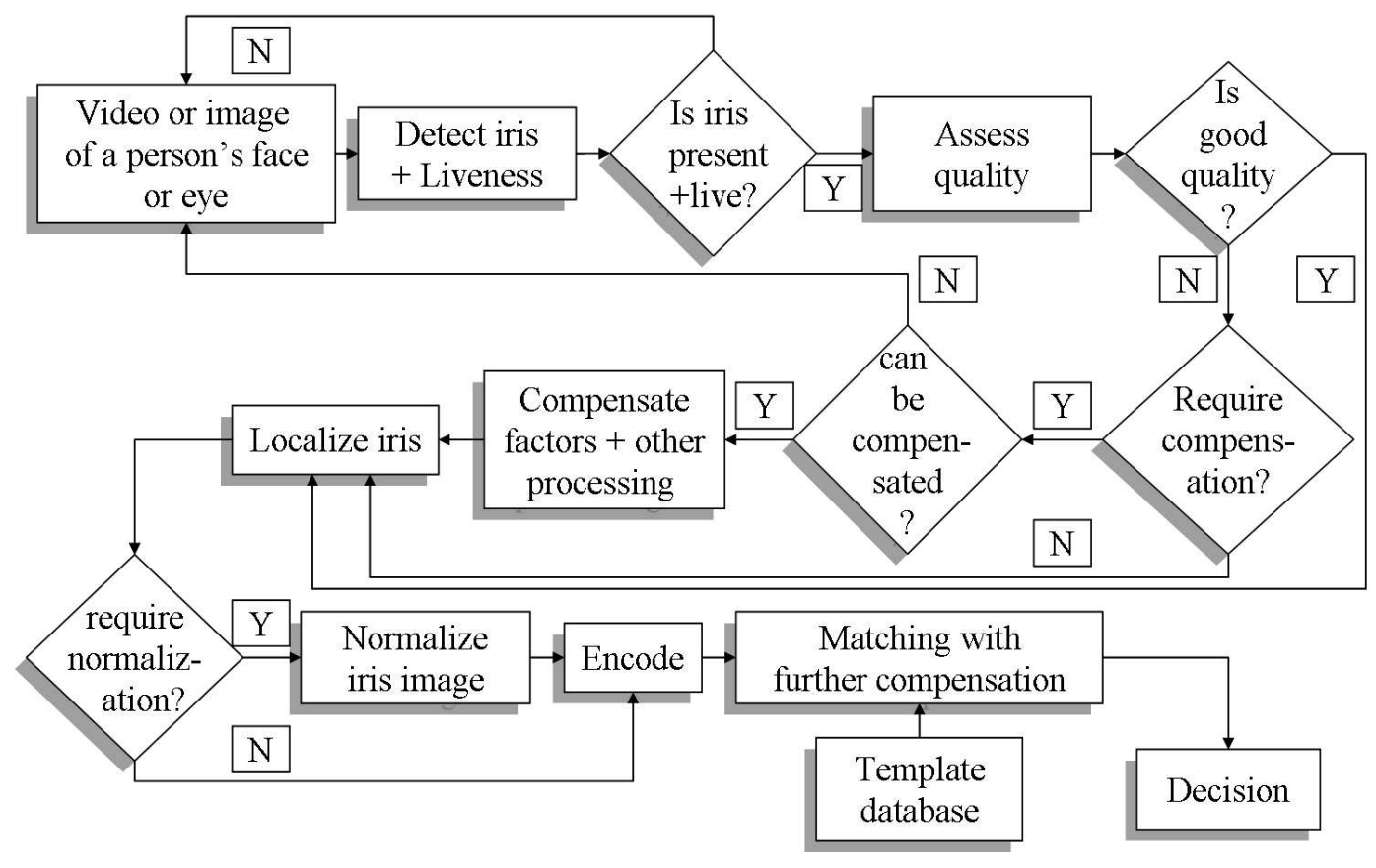

Figure 3.2: A generic Non-Ideal Iris based Recognition System

$$
R=\left(\begin{array}{ccc}
\cos \gamma \cos \beta & \sin \gamma \cos \alpha-\cos \gamma \sin \beta \sin \alpha & \sin \gamma \sin \alpha+\cos \gamma \sin \beta \cos \alpha \\
-\sin \gamma \cos \beta & \cos \gamma \cos \alpha+\sin \gamma \sin \beta \sin \alpha & \cos \gamma \sin \alpha-\sin \gamma \sin \beta \cos \alpha \\
-\sin \beta & -\cos \beta \sin \alpha & \cos \beta \cos \alpha
\end{array}\right)
$$

where $\alpha, \beta, \gamma$ are the angles of rotation with respect to $x, y$, and $z$ axis. A schematic of a non-ideal recognition system is shown in Fig.3.2. Fig.3.3 shows a schematic of a recognition system which deals with off-axis iris images.

\subsection{Estimation of Projective Transformation Ma- trix}

In the estimation of angle of rotation, only one (out-of-plane) axis of rotation is assumed to start with. The best estimate of the angle of rotation would be obtained 


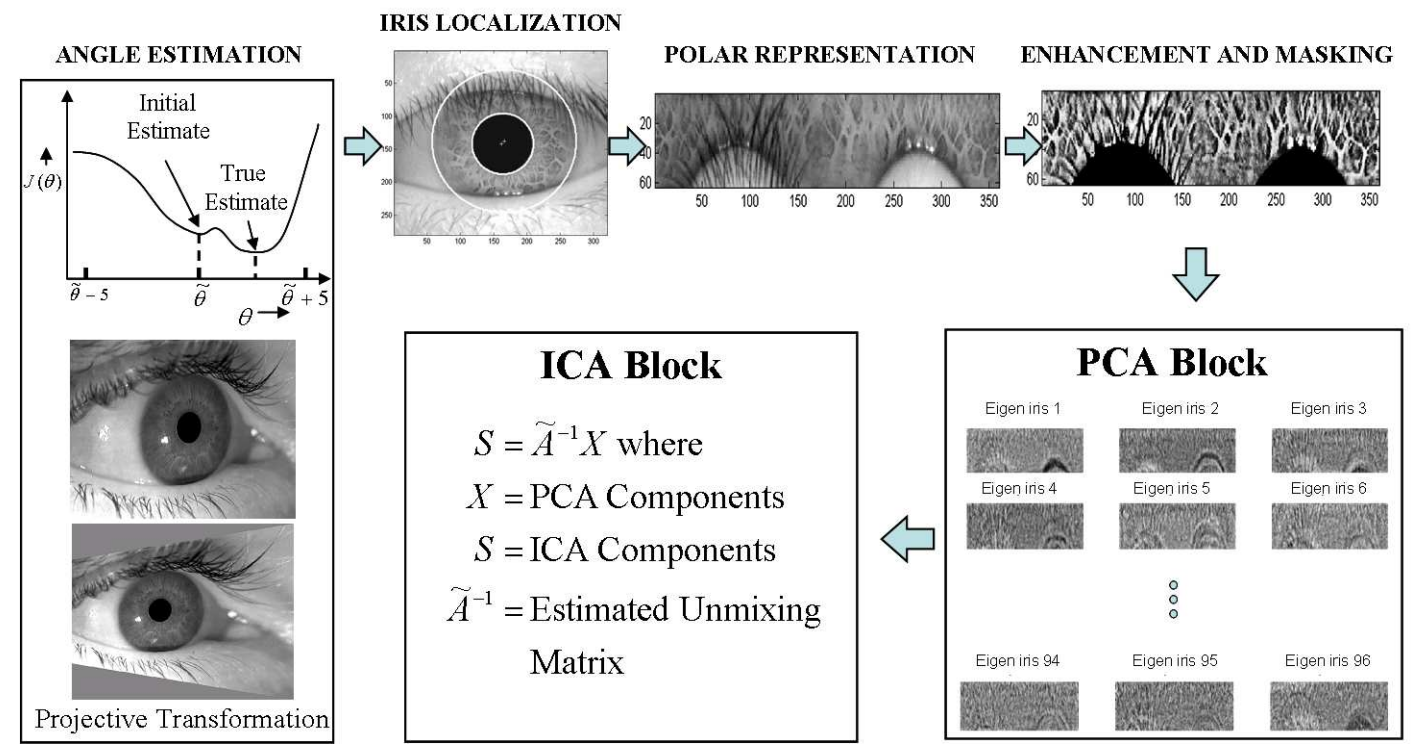

Figure 3.3: A Schematic of a Non-Ideal Iris based Recognition System which deals with off-axis images

by searching all possible values for the angle of rotation. For practical purposes, a few degrees on either side of an initial guess or a multiresolution coarse to fine search can be used [42, 43]. The image is subjected to projective transformation for each probable angle. An objective function is calculated for each transformed image to give a measure of frontal view. The objective function is formulated in such a way that the maxima or the minima of the function is obtained only for an image in frontal view. Here two objective functions are mainly evaluated: (1) the Hamming distance between an ideal frontal view image and a transformed iris image (this is the case when atleast two images are available from the same iris class including atleast one "ideal" iris image) and (2) Daugman's integro-differential operator as a measure of iris circularity (in case if only a single off-angle image is available). We pick the estimates that minimize the Hamming distance and maximize the value of the integrodifferential operator. Equivalently, (1 - Hamming distance) can be maximized. Other approaches can involve ellipse fitting or standard image registration techniques.

The same principle can be extended to two axes of rotation. To estimate these angles of rotation we assume that a rough initial estimate of the angles are available. 
To be more specific, let $\psi_{1}$ and $\psi_{2}$ be two rotational angles $J\left(\psi_{1}, \psi_{2}\right)$ be an objective function that has to be optimized. For each pair of $\left(\psi_{1}, \psi_{2}\right)$ in the range $\psi_{1} \in$ $\left[\psi_{1, \min }, \psi_{1, \max }\right]$ and $\psi_{2} \in\left[\psi_{2, \min }, \psi_{2, \max }\right]$, (i) the off-angle iris image is rotated by using the projective transformation and (ii) the objective function $J\left(\psi_{1}, \psi_{2}\right)$ is calculated as in eq.3.2.

$$
\arg \max _{\left(\psi_{1}, \psi_{2}\right)} J\left(\psi_{1}, \psi_{2}\right)
$$

Once the angles are estimated using the projective transformation, the off-angle image is rotated by the estimated angles to give frontal view image. After this step, any iris recognition algorithm that operates on frontal view iris image can be applied.

Hamming distance requires (1). additional image, and (2). additional processing for both images. Additional processing for the identification case will increase rapidly as compared to the processing required for the circular integro-differential operator. But the use of Hamming distance is optimal in terms of recognition performance. It jointly estimates and gives out a final score in terms of Hamming distance (joint estimation and recognition). If we are using other techniques for encoding, the integrodifferential operator would be more efficient than Hamming distance, though it is only a sub-optimal solution.

\subsection{Ellipse Fitting for Angle Estimation}

Another method for performing this operation is ellipse fitting. This method involves fitting an ellipse to the pupil of the iris. The lengths of the major and minor axes, combined with the angle that the minor axis makes with the horizontal line, gives an estimate of the head tilt for a single image. This does not need any additional image for its operation and is based on the same principle as the integro-differential operator. Though it estimates an angle, the angles can be estimated only upto a sign value, that is, though the estimate of the angle of rotation is known, the direction is not known, which would involve some additional processing, say with an operator which can give a measure of circularity (circular integro-differential operator) of the pupil after transformation in both positive and negative directions. This information 
can be used to obtain a frontal view image.

Care needs to be taken when we directly normalize the inner and outer boundary by fitting ellipses. This is because of the nature of the transformation (projective) the image undergoes, which in most cases would require non-linear sampling as against the usual linear sampling during normalization and should be wisely carried out according to the conditions at hand. Once transformed into frontal view, linear sampling should give reasonably good results.

There are numerous techniques to perform ellipse fitting. One efficient method for fitting ellipse to a close-up iris image is presented in [44]. A generalization of Daugman's integro-differential operator to an ellipse would lead to finding the maxima of the function

$$
\max _{\left(a, x_{0}, y_{0}, e, \phi\right)}\left|G_{\sigma}(a) * \frac{\partial}{\partial a} \oint_{a, x_{0}, y_{0}, e, \phi} \frac{I(x, y)}{p} d s\right|
$$

where $*$ denotes convolution, $G_{\sigma}(a)$ is a smoothing function such as a Gaussian of scale $\sigma, a$ is the semi-major axis of the ellipse, $\left(x_{0}, y_{0}\right)$ the centre of the ellipse, $e$ being the eccentricity and $\phi$ is the angle of the minor axis of the ellipse. Here, $p$ is the perimeter of the ellipse. In the continuous space, various approximations including GaussKrummer series, Ramanujan's approximations, etc.[45] exist though not required for discrete implementation. Projective transformation can be applied after estimating both the angles of rotation and the sign of direction (that is, positive or negative). If projective transformation needs to be eliminated, the doubly dimensionless polar coordinate system must be sampled non-linearly if the iris is assumed to be a planar object. 


\section{Chapter 4}

\section{Proposed Encoding Techniques}

The general block-diagram of the system is shown in Fig.4.1. Below is a brief description of the two encoding blocks.

\subsection{Encoding Using PCA}

The idea of using PCA for biometric applications was motivated by the work of Sirovich and Kirby[46] for efficient representation of face images. It was shown that a large number of face images could be reasonably well approximated by storing a few weights each corresponding to a standard set of trained bases, for each face image. Later, Turk and Pentland[47] went on to use the variations in the weights to identify faces. The idea is the same, except that we use iris images. Though the task seems simple, there are other factors that need to be considered. The distortions and transformations undergone by an iris image are significantly different from that of a face image. There are no well known set of features across all iris images which seem to remain fixed. To start with, an approach as described in [47, 48] is presented. Later, modifications are made to accommodate various distortions. The main idea is to find a set of vectors that contribute to significant variation across training images. In this section, we briefly characterize the PCA method adopted to perform iris encoding (for details on PCA see $[47,48]$ as well as the appendix). A typical PCA algorithm operates in two modes: training and testing as shown in Fig.4.2 and Fig.4.3. During 


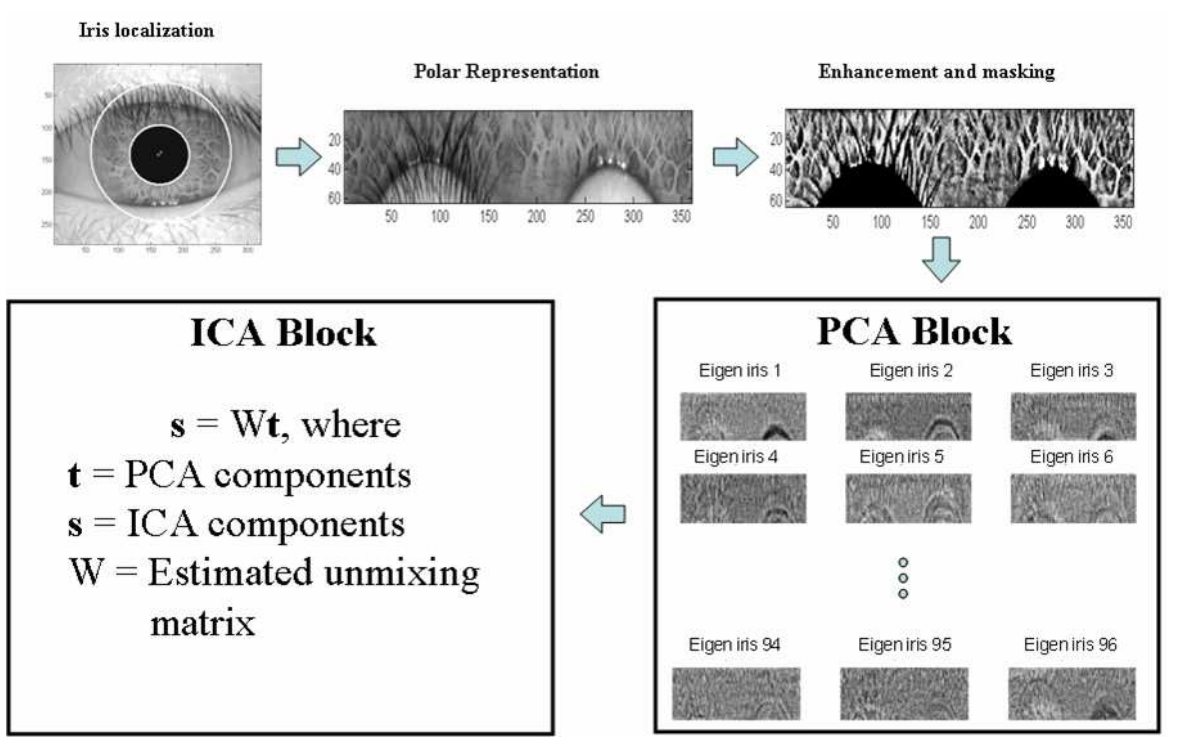

Figure 4.1: Block-diagram of the system implementing PCA/ICA encoding techniques for iris.

the training mode, the principal components are extracted using labeled training data. During the testing mode, the performance of the iris identification system is evaluated. Let $\mathrm{I}(x, y)$ be an iris image of appropriate size, say $64 \times 360(n \times m)$ which is vectorized into a one dimensional vector by placing one column below the other. Now the size of this vector is $(n m) \times 1$. Let $M$ be the number of iris classes. Suppose that a training set $\mathbf{X}_{\mathbf{1}}, \mathbf{X}_{\mathbf{2}}, \ldots, \mathbf{X}_{\mathbf{n}}$, a sequence of normalized and preprocessed iris images(vectorized) indexed in accordance with iris class, is available. This data are used to form the scatter matrix

$$
\Sigma=\frac{1}{M-1} \sum_{i=1}^{M}\left(\mathbf{X}_{m}-\overline{\mathbf{X}}\right)\left(\mathbf{X}_{m}-\overline{\mathbf{X}}\right)^{T}=A A^{T}
$$

where $\overline{\mathbf{X}}$ is the empirical mean. Since $\Sigma$ is positive definite and symmetric, it can be decomposed using the eigenvalue decomposition method known also as the Karhunen-Loeve expansion. Thus

$$
\Sigma=\mathbf{Q} \Lambda \mathbf{Q}^{T}
$$




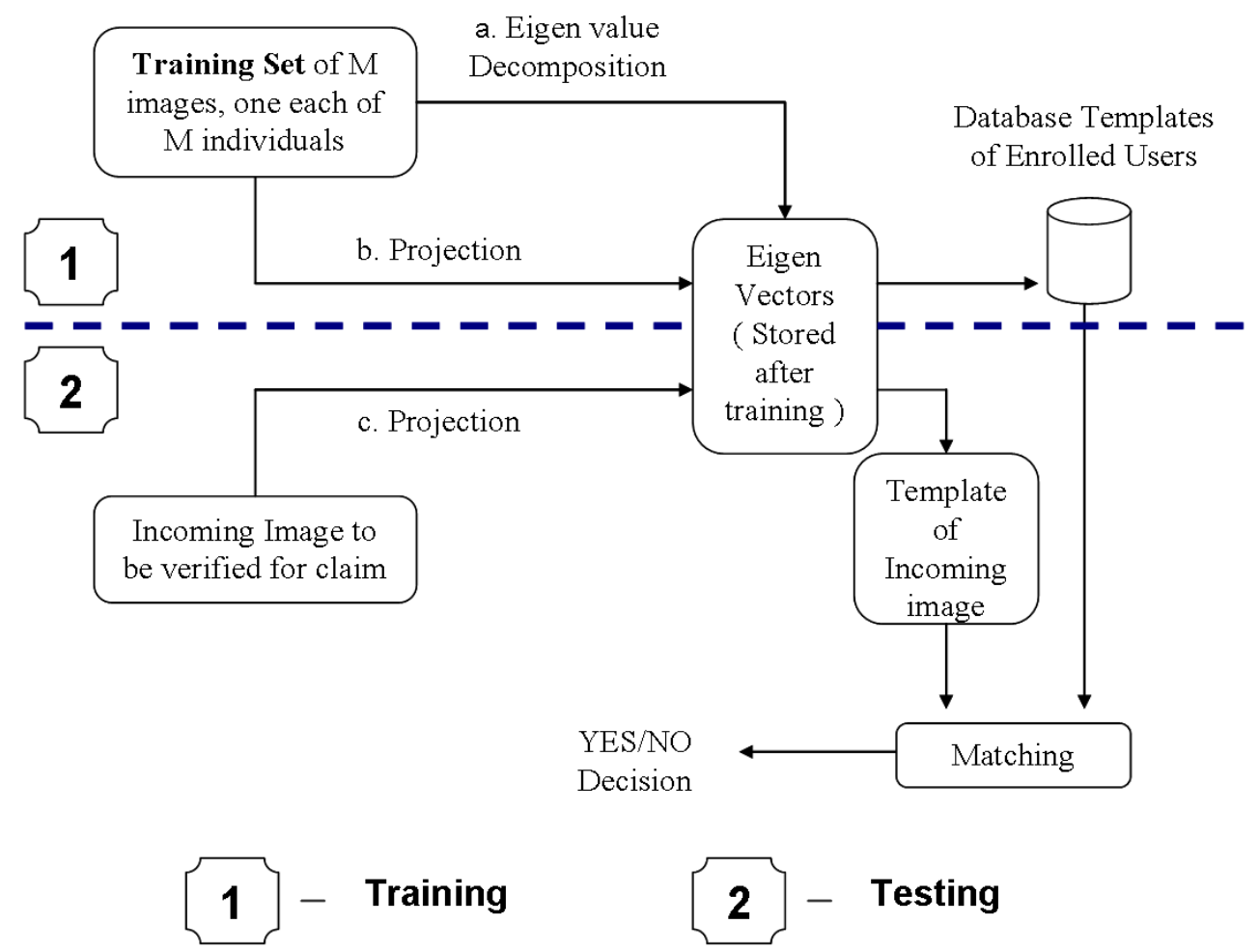

Figure 4.2: Schematic of testing scenario 1

where $\Lambda$ is the diagonal matrix of the eigenvalues of arranged in decreasing order and $\mathrm{Q}$ is the orthogonal matrix whose columns form the eigenvectors of $\Sigma$ (the columns can be chosen to be orthogonal). Geometrically, the eigenvectors are the basis vectors of the transformed coordinate system. In practice, the smallest eigenvalues of $\Sigma$ are disregarded, and new matrix $\widetilde{\mathbf{Q}}$ with vector columns corresponding to the essential eigenvalues is formed. Thus the new transformed space has smaller dimension than the original space (data compression concept). For iris images, as will be demonstrated later, the compression is poor.

The above mentioned procedure in practise is however made difficult due to the dimensionality of the covariance matrix $\Sigma$ which would be of size $(\mathrm{nm}) \times(\mathrm{nm})$. Diagonalizing such as huge matrix and finding its $n m$ eigenvalues and eigenvectors by itself 
is now rendered difficult. In order to overcome this hurdle, properties of eigenvalues and eigenvectors were efficiently used in [47] to make the solution computationally feasible. The number of meaningful eigenvalues would only be $M-1$ though the dimensionality of the space is $n m$. Hence in this case, we can solve a $\mathrm{M} \times \mathrm{M}$ matrix for eigenvalues and eigenvectors instead of a $(n m) \times(n m)$ matrix from which we can determine $M-1$ meaningful eigenvectors of the huge covariance matrix. Let $\mathbf{v}_{i}$ be the eigenvectors of $A^{T} A$. Then,

$$
A^{T} A \mathbf{v}_{i}=\mu_{i} \mathbf{v}_{i}
$$

Pre-multiplying both sides by $A$, we get

$$
A A^{T}\left(A \mathbf{v}_{i}\right)=\mu_{i}\left(A \mathbf{v}_{i}\right)
$$

Hence, $A \mathbf{v}_{i}$ are the eigenvectors of $A A^{T}$. This makes calculation of meaningful eigenvectors easy. The eigenvectors of $A^{T} A$ are calculated and are pre-multiplied with $A$ to get the eigenvectors of $A A^{T}$.

To test the algorithm, each vector in the testing set is further projected onto the axes of the new transformed space, and the coefficients of projections are collected in the vector of features, $\mathbf{W}$. To measure the distance between two projected iris images, we involve two distances: (i) Euclidean and (ii) Hamming. To involve the second measure, we quantize the values of individual coefficients in the vector $\mathbf{W}$ to "1" or "0" if the feature value is greater than zero and less than or equal to zero, respectively. In order to take rotation into consideration, one of two segmented and enhanced iris images is rotated systematically on either direction (up to a few degrees) and templates are extracted for each rotated version of the image. Let $\mathbf{W}_{1}=\widetilde{\mathbf{Q}}^{T} \mathbf{Y}_{1}$ and $\mathbf{W}_{\mathbf{2}}=\widetilde{\mathbf{Q}}^{T} \mathbf{Y}_{\mathbf{2}}$ be two PCA coefficient vectors corresponding to two distinct normalized and preprocessed iris images $\mathbf{Y}_{\mathbf{1}}$ and $\mathbf{Y}_{\mathbf{2}}$ from the testing set. Then to compensate for rotation the following minimization step is performed

$$
\min _{\theta \in\left[-\theta_{\max }, \theta_{\max }\right]} d\left(\widetilde{\mathbf{Q}}^{T} \mathbf{Y}_{\mathbf{1}}, \widetilde{\mathbf{Q}}^{T} \mathbf{Y}_{\mathbf{2}}(\theta)\right)
$$




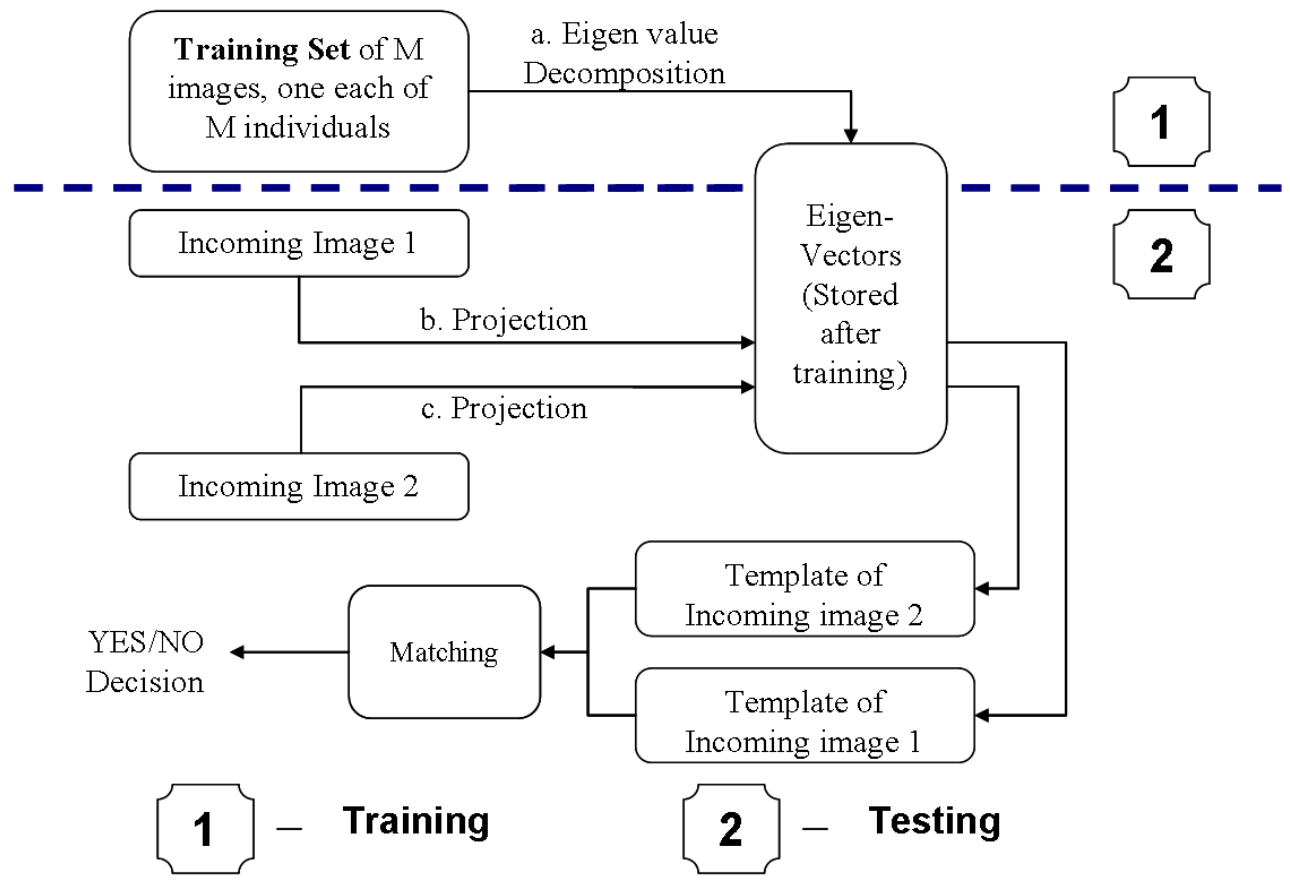

Figure 4.3: Schematic of testing scenario 2 
where $d($.$) denotes the Euclidean or Hamming distance between two projected iris$ images and $\widetilde{\mathbf{Q}}$ is the matrix composed of eigenvectors corresponding to only essential eigenvalues. Note that for normalized images the rotation operation reduces to the cyclic shift operation of the second image with respect to the first image.

\subsection{Following up with ICA}

ICA is a subspace analysis technique. It aims to find a set of independent sources that capture the underlying randomness of the observed signals. ICA has been applied to iris analysis in [29, 30, 31]. In those works, randomly selected patches of a small size from iris images form a training set. This training set is then processed using PCA to reduce its dimensionality and decorrelate components, before applying ICA locally. In theory, whitening before ICA is not a necessary step. Though, whitening aids estimation of independent components. The training templates available after applying PCA form the input to the ICA block. An unmixing matrix, which represents the ICA basis vectors, is estimated from these PCA input templates by minimizing the mutual information (a measure of dependence) between transformed components in ICA space. Previous ICA algorithms [29, 30, 31] do not take rotation (image alignment) into account. While this step seems unnecessary in [29, 30, 31], compensation for a rotation uncertainty is a critical step for our non-ideal iris application. In this work, we use ICA as a follow up encoding method in anticipation that it will pick individual fine features present in iris images and thus will improve the performance of PCA method. Unlike previous ICA algorithms used for iris recognition, we do not divide iris images into patches during the training step but rather use the entire iris image to estimate the unmixing matrix and further to extract ICA components. Denote by $\widetilde{\mathbf{X}}_{\mathbf{1}}, \widetilde{\mathbf{X}}_{\mathbf{2}}, \ldots, \widetilde{\mathbf{X}}_{\mathbf{n}}$ a sequence of preprocessed, normalized, and whitened iris images indexed by their class. During the training mode we assume that each class is represented by a single iris image. It can be easily generalized to a multi-image case. ICA is a blind source-separation method. It assumes that observed data can be represented as a linear combination of a number of independent signals. The unknowns are the mixing coefficients and the independent input signals. Let $\widetilde{X}$ be a 
matrix with vector columns given by $\widetilde{\mathbf{X}}_{\mathbf{1}}, \widetilde{\mathbf{X}}_{\mathbf{2}}, \ldots, \widetilde{\mathbf{X}}_{\mathbf{n}}$ and $\mathbf{S}$ be a matrix composed of unknown independent input signals arranged in columns. Then ICA assumes the following forward model

$$
\widetilde{\mathbf{X}}=\mathbf{A S}
$$

where $\mathbf{A}$ is the unknown mixing matrix. As argued in [49], the results of linear mixing of non-Gaussian signals are more Gaussian than the input signals. Then to estimate the mixing matrix $\mathbf{A}$ and one of the components of $\mathbf{S}$, one has to define a measure of non-gaussianity. One of the theoretically sound criteria is the maximization of the negentropy given by

$$
J=H_{\text {Gaus }}\left(\mathbf{A}^{-1} \mathbf{X}\right)-H\left(\mathbf{A}^{-1} \mathbf{X}\right)
$$

where $H_{\text {Gaus }}$ is the entropy of the data under the assumption that data are Gaussian distributed and under the constraint of the same covariance matrix for the distributions in $H_{\text {Gaus }}$ and in $H$ (see [49] for more detailed explanation). Once the mixing matrix and one of the input signals are estimated, the remaining input signals can be obtained by invoking the Gram-Schmidt orthogonalization procedure. To deal with empirical case, (2) is approximated by expressions involving empirical moments. To test the performance of ICA method, we use testing data that are different from the training set. Similar to the case with PCA encoding, we apply two distance measures to perform matching (i) Euclidean and (ii) Hamming distances. This is done for the purpose of performance comparison. To overcome the effect of rotation during testing, we project each rotated version of the two images into PCA space and then into ICA space and obtain the minimum score between the templates of the different rotated versions. Let $\mathbf{W}_{\mathbf{1}}=\mathbf{S} \widetilde{\mathbf{Q}}^{T} \mathbf{Y}_{\mathbf{1}}$ and $\mathbf{W}_{\mathbf{2}}=\mathbf{S} \widetilde{\mathbf{Q}}^{T} \mathbf{Y}_{\mathbf{2}}$ be two vectors of ICA coefficients corresponding to two distinct normalized, preprocessed iris images $\mathbf{Y}_{\mathbf{1}}$ and $\mathbf{Y}_{\mathbf{2}}$ from the testing set. The following minimization procedure is applied to compensate for rotation

$$
\min _{\theta \in\left[-\theta_{\max }, \theta_{\max }\right]} d\left(\mathbf{S} \widetilde{\mathbf{Q}}^{T} \mathbf{Y}_{\mathbf{1}}, \mathbf{S} \widetilde{\mathbf{Q}}^{T} \mathbf{Y}_{\mathbf{2}}(\theta)\right)
$$


where $d($.$) denotes the Euclidean or Hamming distance between two projected iris$ images and $\widetilde{\mathbf{Q}}$ is the matrix of essential eigenvectors introduced in Sec. 2.2. For the results presented here, the FastICA package was used [50]. 


\section{Chapter 5}

\section{Results}

The results are presented in the same order in which the proposed non-ideal system would operate. First, the results for estimation techniques are presented. Secondly, results for encoding (tested on a database of frontal view images)is presented. This is followed by the performance of the proposed system. These are followed by various studies on the proposed encoding schemes, as well as other non-ideal factors.

\subsection{Performance of Estimation techniques}

Estimation of the projective transformation matrix, calculated in terms of the angles of rotation is carried out on WVU's off-angle database. The database now has 200 classes of irises from a hundred users with four images per iris class, two from frontal view, one from 15 degrees and the other from 30 degrees. Some examples of off-angle images are shown in Fig.5.13. In this section, some examples of estimated frontal view images are shown along with their respective objective functions. It can be seen that the objective function gives a good estimate of a frontal view image.

It should be noted that the iris images rotated at say 15 or 30 degrees can sometimes require fewer degrees of compensation in order for the pupil to become circular. This can be due to several reasons.

1. The head tilt and tilt of the iris may not be the same. 

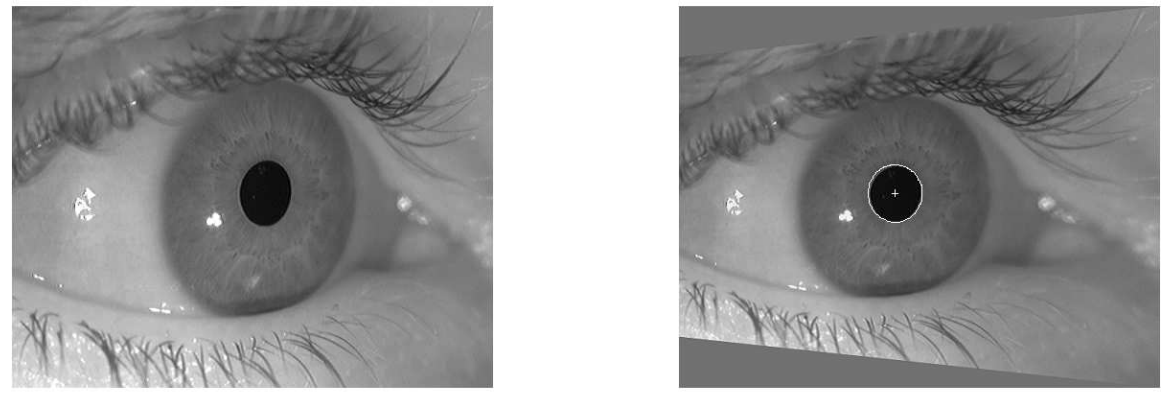

(a) Original Image

(b) Transformed Image

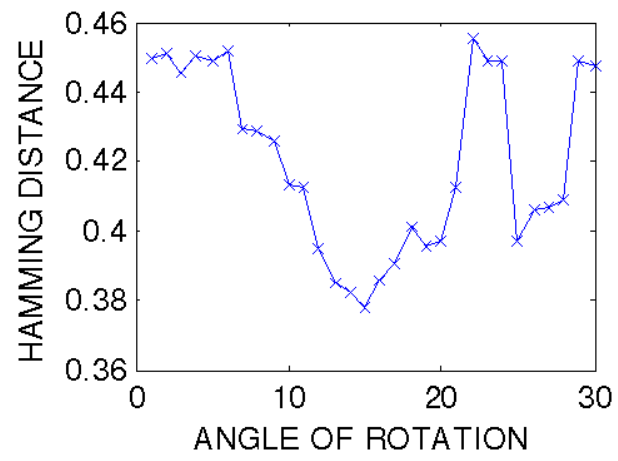

(c) Objective function for various values of estimates

Figure 5.1: Estimating Off-Angle Using Projective Transform with one axis of rotation using Hamming Distance as criterion 


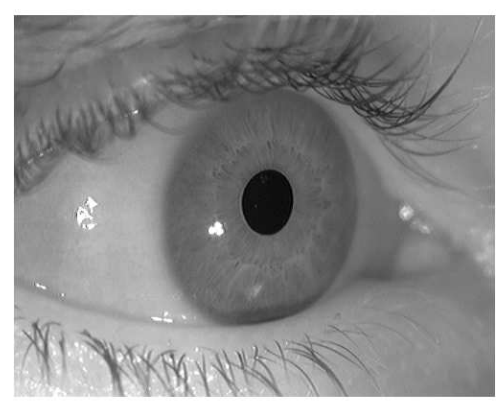

(a) Original Image

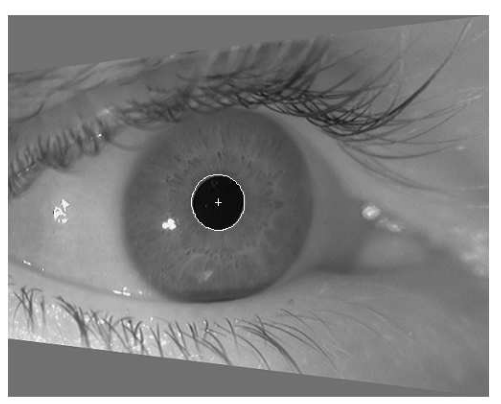

(b) Transformed Image

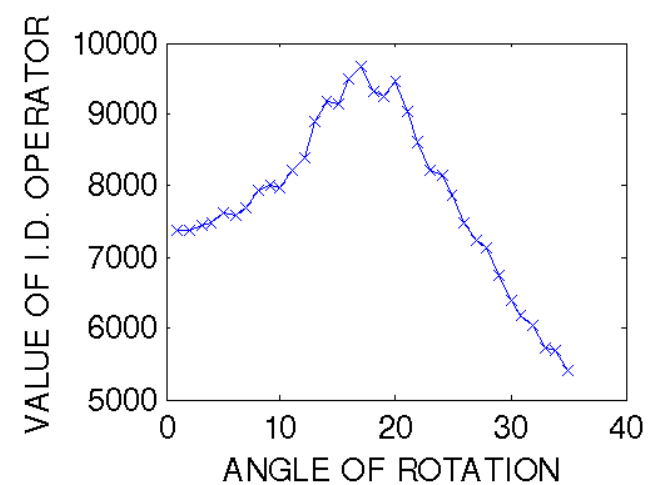

(c) Objective function for various values of estimates

Figure 5.2: Estimating Off-Angle Using Projective Transform with one axis of rotation using Integro-differential operator 


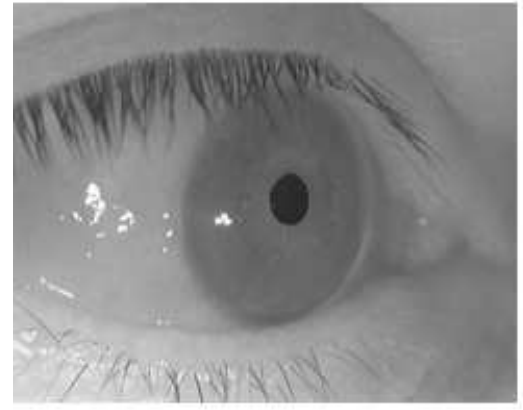

(a) 30 degree image

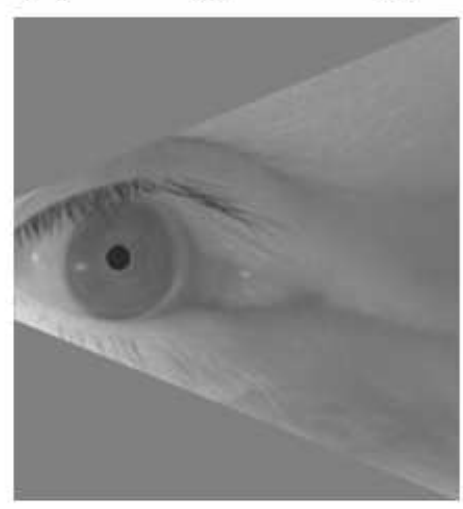

(b) Rectified image

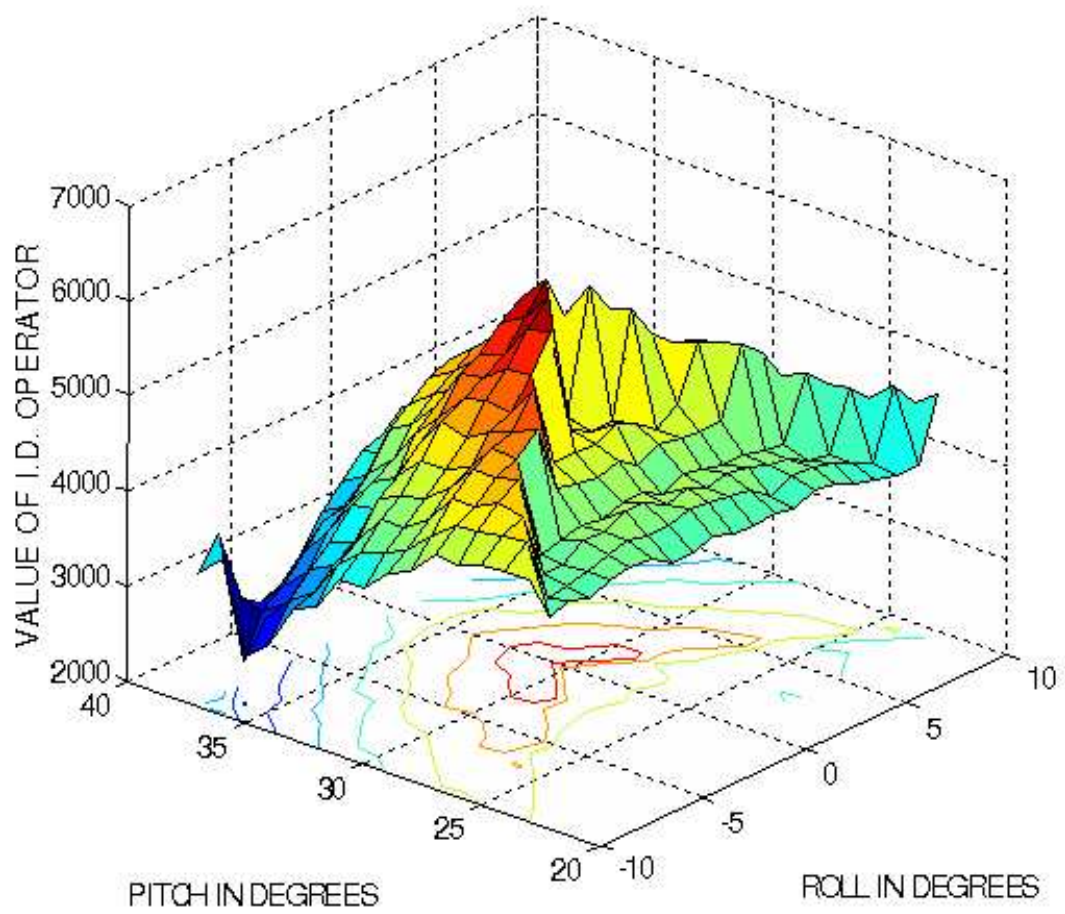

(c) Value of objective function for various angles

Figure 5.3: Estimating Off-Angle Using Projective Transform with two axes of rotation 


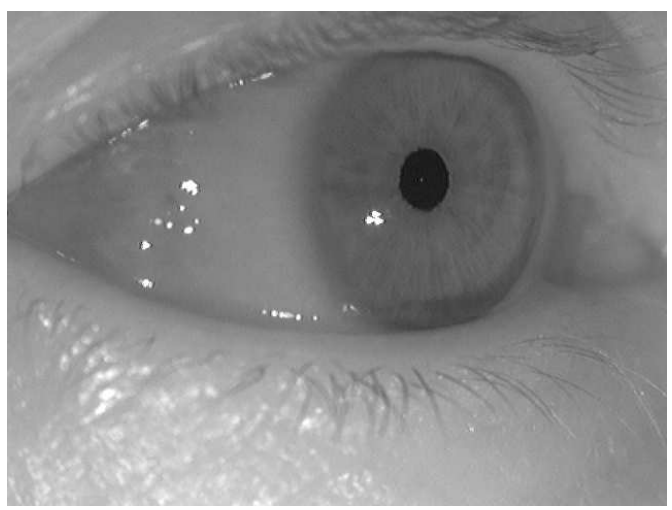

(a) Original image 1

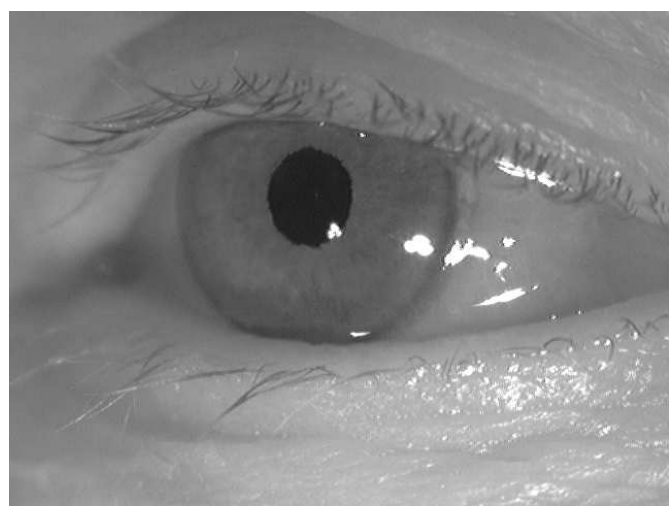

(c) Original image 2

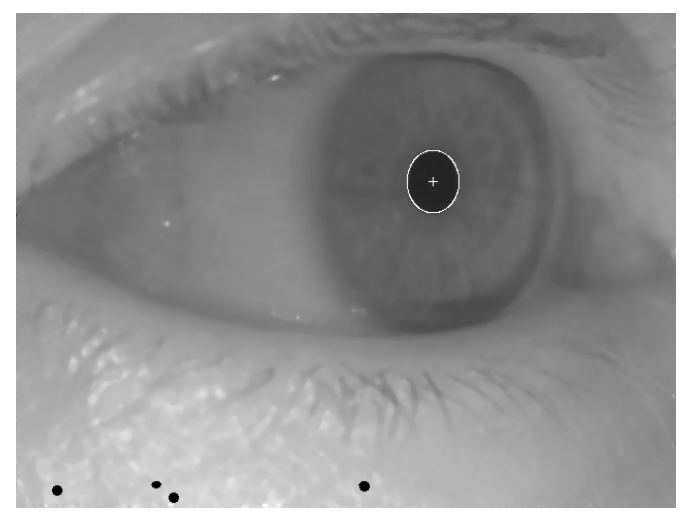

(b) Ellipse fitted to original image 1

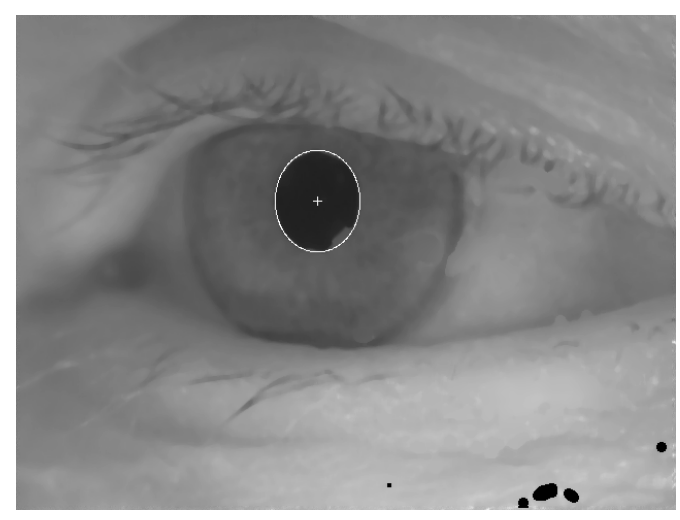

(d) Ellipse fitted to original image 2

Figure 5.4: Estimating ellipse parameters using the elliptical integro-differential operator 
2. The effect due to aqueous humor and cornea which lies between the iris surface and the image capture device.

3. Other distortions due to the capture device.

Even if these effects are compensated, information is either introduced or lost due to interpolation or sampling during projective transformation even when greatest care is taken. This may not pose a great problem from a recognition point of view if handled carefully. Other challenges involve the determining whether the iris is tilted either in the positive or negative direction. This information will help reduce computational complexity as well as bad estimates. The entire image of the "eye" may not be always available.

\subsection{Results for Encoding of Frontal View Images}

All the experiments in this section are performed on the CASIA dataset provided by the Chinese Academy of Sciences [51]. The CASIA dataset contains iris images of 108 iris classes with 7 images per iris. Some images in this dataset are strongly occluded, blurred, and defocused. Sample images from CASIA datasets are shown in Fig.2.1. Experiments were also conducted on a smaller collection of 22 iris classes at our labs in WVU which are presented later. As a baseline curve, we use the receiveroperating characteristic (ROC) for modified J. Daugman's system [52]. The results of the modified J. Daugman's system implementation are shown in Fig.5.7 (black solid line). In our experiments, global PCA method when applied to iris images, extracted 99 essential eigenvalues to give good performance. The value of 99-th eigenvalue is significantly larger when compared with the value of the first eigenvalue. This resulted in a poor compression. This also emphasizes the fact that individual iris is rich in information.

\subsubsection{Compensation for Rotation}

We first demonstrate the effect of rotation on the performance of PCA and global ICA encoding methods. Fig.5.6 displays two sets of histogram distributions of genuine and 
imposter Euclidean distance scores obtained using the data from the CASIA dataset. The left and the right panels in Fig.5.6 show the results for iris verification system implementing PCA encoding technique without and with compensation for rotation, respectively. Since iris images during processing are transformed to have a pseudopolar representation, the angle values used to optimize the performance are measured in the number of pixels. Each image is normalized such that one pixel in the normalized image corresponds to one degree in the original image. The range of angles used during these experiments was set to $[-10,10]$. Similar results were obtained for encoding with ICA technique. The ROC curves demonstrating the effect of rotation are shown on the left and right panels in Fig.5.7 for PCA and ICA encoding techniques, respectively. The results are displayed both for Euclidean (ED) and Hamming (HD) distances. One can conclude that the compensation for head tilt (rotation) leads to a substantial improvement of performance. Only for results involving both Hamming distance and ICA, rotation operation is performed during the PCA step. The lowest scoring PCA template is projected on ICA space. Two scenarios: The performance of the system in Fig.4.1 is further evaluated using two different scenarios. In the first scenario, we formed two sets of iris images, training and testing, from the CASIA database. Each set consisted of one image from 100 different irises. The training set was formed from the third image of first 100 irises, while the testing set was formed from the second image of the same irises. In the second scenario, we used the training only to extract eigenvectors and discarded all training set images. During testing, PCA components (the first technique) or ICA components (the second technique) of each image from the test data set were obtained and compared against PCA/ICA components of the other images in the testing set. Thus, the second scenario can be viewed as a "blind" testing. The ROC curves for both scenarios are shown in Fig.5.5 . The left panel in Fig.5.5 demonstrates the results when Hamming distance is used as the matching score distance. The right panel demonstrates similar results for the case when Euclidean distance is applied to calculate the matching scores. As expected, the results of testing under the "blind" scenario are slightly degraded (both for PCA and ICA) compared to the results obtained under the first scenario.

The advantage of the "blind" scenario is in its flexibility. The coefficients forming 


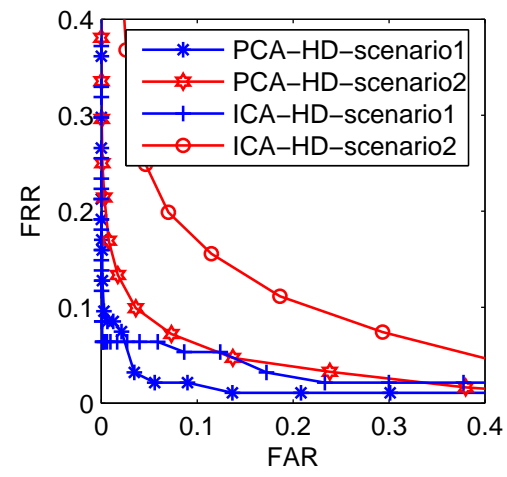

(a) Hamming Distance

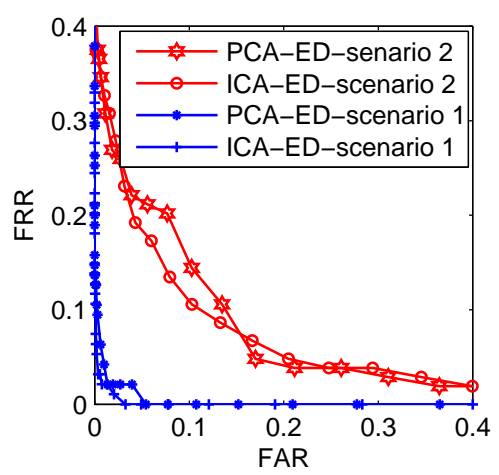

(b) Euclidean Distance

Figure 5.5: Performance in different scenarios

PCA and ICA vectors need not be stored in a user database.

\subsubsection{Occlusion, Masking and Performance}

The presence of extraneous information such as eyelids and eyelashes along with the highly unique iris structure may influence the performance of the algorithms used. Hence, it may be beneficial to remove such "outside" information in order to assess the performance of the system based only on "iris information". Since the algorithms mentioned above use global information and training is done on normalized images, it is possible that the algorithm considers part of outside information as possible features that will be used to distinguish between individuals. For effectively handling such a situation, local feature extracting methods use occlusion masks.Occlusion masks are of the same size as that of the normalized iris images. It contains zeros in the locations where occlusion is present in the iris image and ones in the place where iris structure is present.

To incorporate such a flexibility in the global feature extractor such that there is minimal contribution to recognition from eyelashes and eyelids, there are some simple ways that one would immediately come up with. One is to calculate the masks of all individual eyes and overlay each mask obtained, on the normalized iris images during testing (by multiplying the normalized image with the mask image, pixel by 


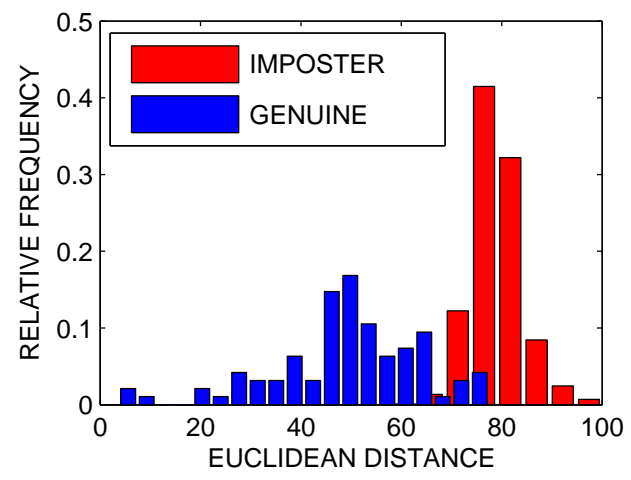

(a)

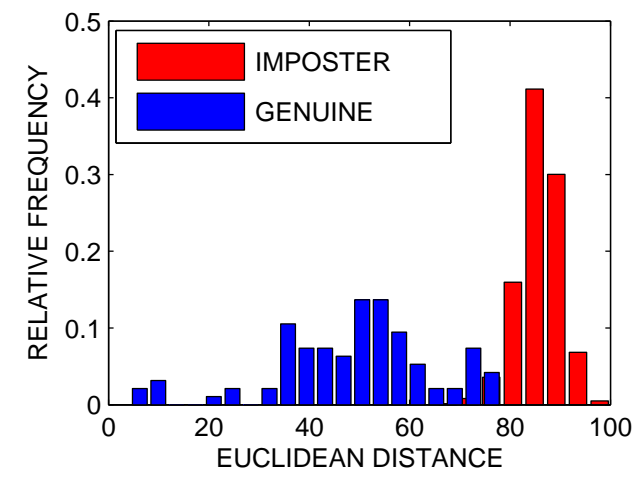

(b)

Figure 5.6: Effect of rotation on matchscores, (a).Histogram before compensation, (b).Histograms after compensation

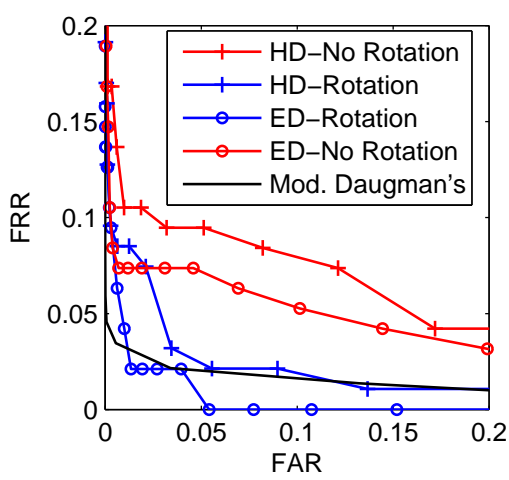

(a)

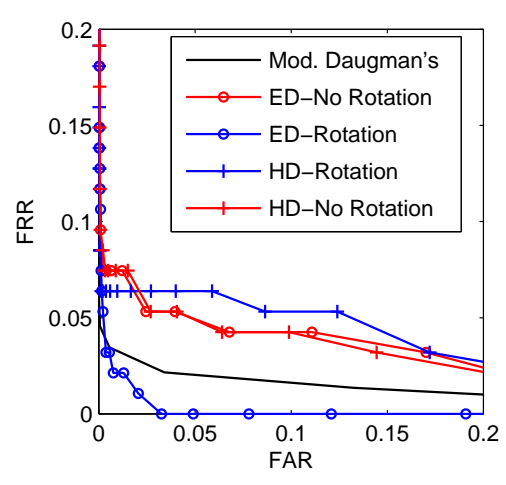

(b)

Figure 5.7: PCA/ICA results for various simulations, (a).ROC curves for PCA, (b).ROC curves for ICA 
pixel). Hence, in all pixel locations which initially consisted of gray scale values from occlusions, it will now be some constant value (zero in this case). But this would mean that different training images (as well as testing images) would have different masked areas. There is again is a possibility that the boundary between the masked area and the iris texture region could again be considered as a feature by the feature selection algorithm as the boundary is of different shapes in different training images. This would totally defeat the purpose of what the mask was meant to do in the first place. A better way would be to have a common mask for all training images (if not the testing images). By this we make the region covered by the common mask, common across all the users and hence redundant. Since all these values are constant (zero in this case), it would not affect the final matching score.

Since it is now decided that a common mask would be used, it can be constructed in two ways. One is to perform an "AND" operation on all the individual masks. But even if a single or couple of masks have extremely high occlusion, the final area of iris texture under consideration for all the training and testing images will be very small. To discard such images from training will be to train the system on a biased set of users. Hence, to deal with such a situation and to strike a balance between including too much occlusion and losing too much information, a standard mask that covers probable iris regions is generated. This is not dependent on individual masks of any of the iris images but based on the knowledge that many times during capture of an iris image, there is some amount of occlusion from both upper and lower eyelid.

Now the experiments are performed with such as standard mask and the masked images. The eigenvectors and ICs for the training set are shown in Fig.5.9 and Fig.5.10. The performance of such a system is compared with the previous version without mask in Fig.5.11 in terms of their respective ROCs. Here, we see that the PCA algorithm has a degraded performance than before but ICA algorithm performed after PCA improves significantly with standard masks applied. The improvement in performance of ICA followed by PCA indicates that this particular kind of algorithm captures fine features better than the more dominant structure information, while PCA tries to capture structure information at a low frequency better than the finer features. The reader is cautioned to be extremely cautious in selection of such standard 


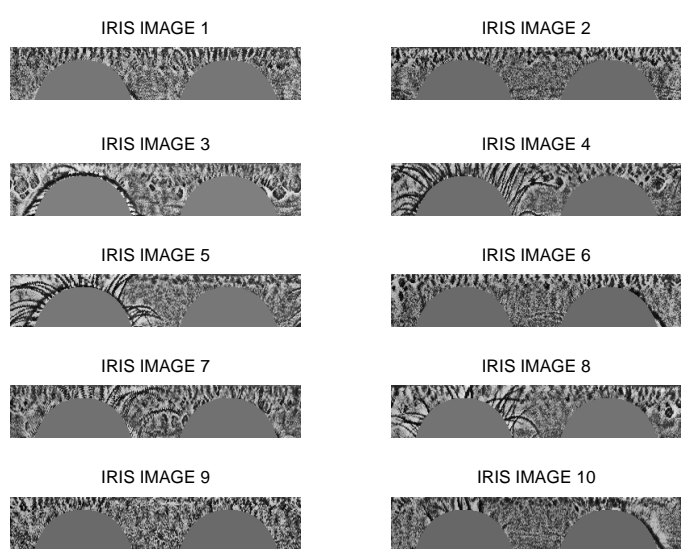

Figure 5.8: Some examples of masked images

mask, especially when specular reflections are a part of the acquisition device.

The masking procedure makes the results more robust. It also gives an idea of the kind of features that contribute more to recognition.

\subsection{Performance of the Non-ideal Recognition sys- tem}

For considering the effect of view on performance, testing is done on twenty seven iris classes from the WVU's off-angle iris image dataset. Distances based on global ICA algorithm are calculated for three different sets of images. The three sets are

1. Two zero degree images per iris class.

2. One zero degree image and one fifteen degree image per iris class.

3. One zero degree image and one thirty degree image per iris class.

The results shown in Fig.5.14 were obtained by performing projective transformation at an angle estimated initially during collection of the images. Only one axis of rotation is considered. The axis of rotation can be assumed to lie on either the left 


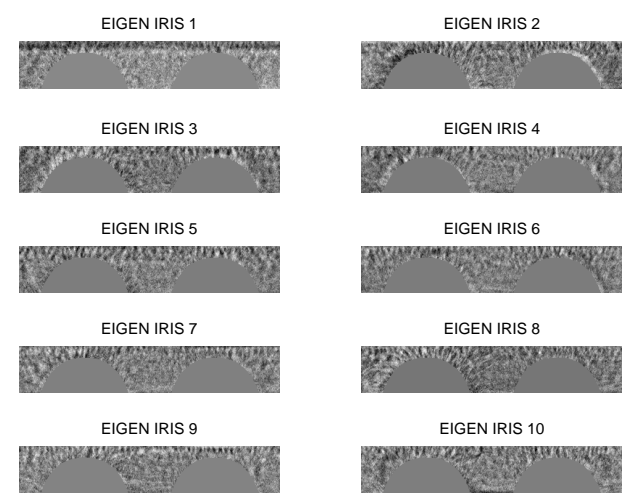

Figure 5.9: Some examples of Independent irises

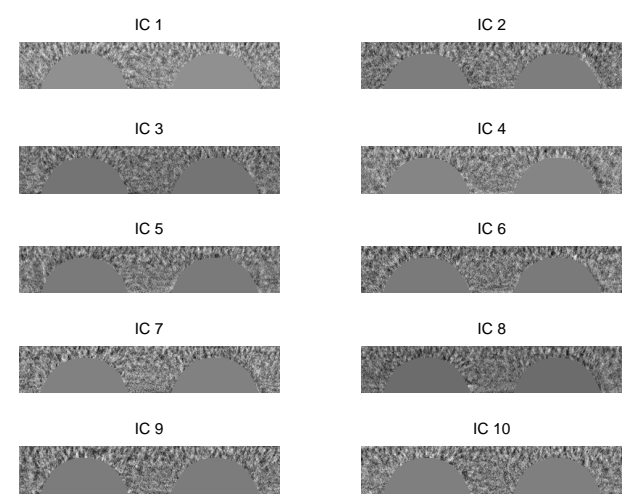

Figure 5.10: Some examples of Eigen irises

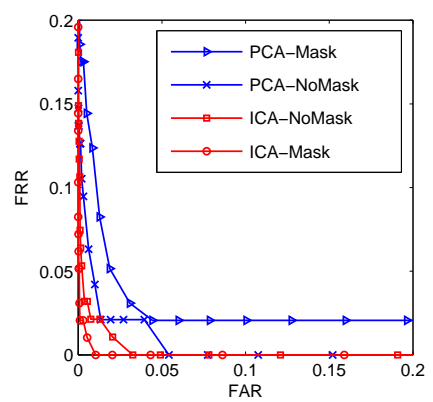

Figure 5.11: Effect of applying a unified mask to reduce interference from eyelids 


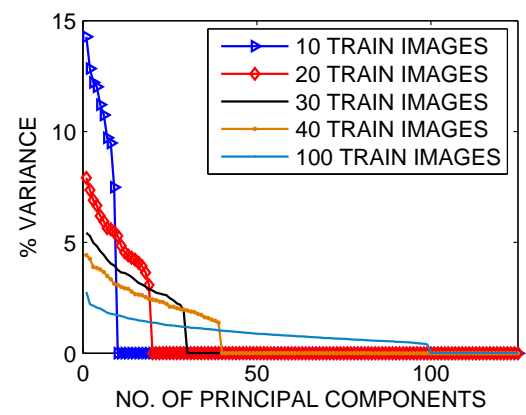

(a)

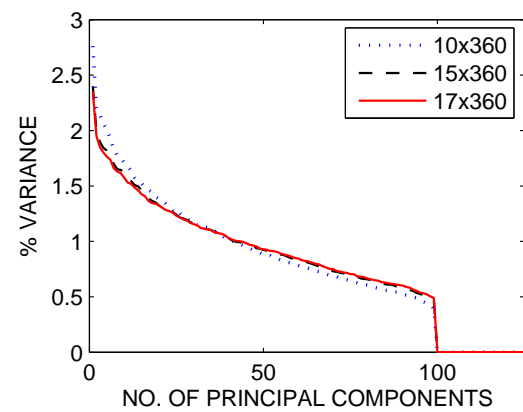

(b)

Figure 5.12: Percentage variance contributed by each eigen vector to the total variance as a function of (a) Varying resolution of training image with constant number of training templates (b) Varying number of templates for constant resolution

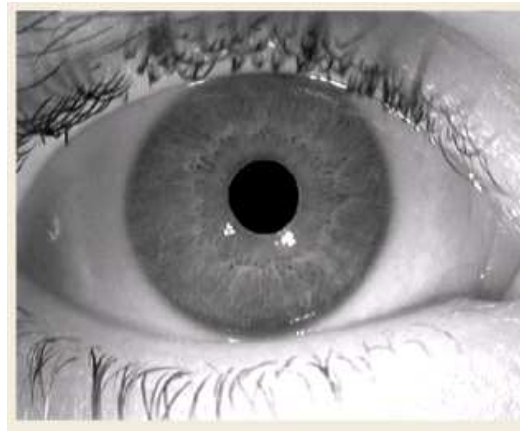

ANGLE: 0 DEGREES

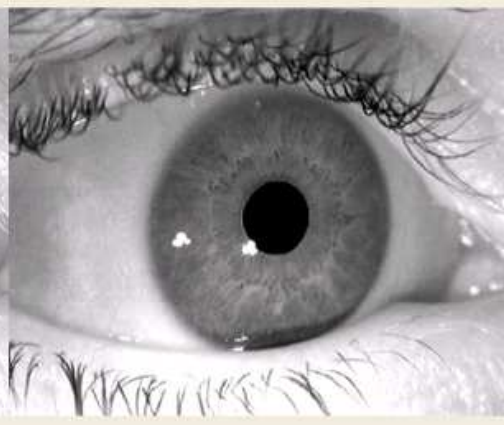

ANGLE: 15 DEGREES

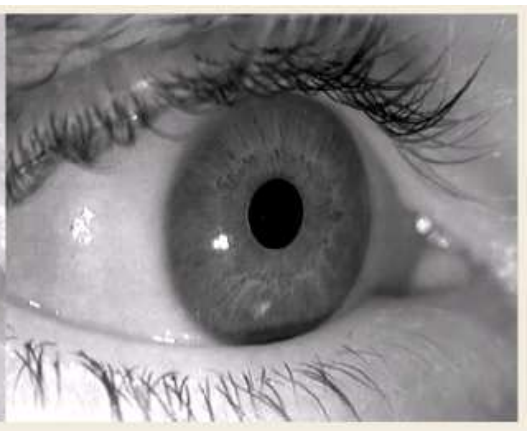

ANGLE: 30 DEGREES

Figure 5.13: Three samples of a single user from an off-axis dataset collected in our labs at WVU 
edge, right edge, or center of the image. The true axis of rotation is unknown. Here it was assumed to be at the centre of the image.

The results presented in Fig.5.14 were performed on the above mentioned database from WVU. It shows ROCs for different combinations of training and testing when using the initial estimate as the true estimate.

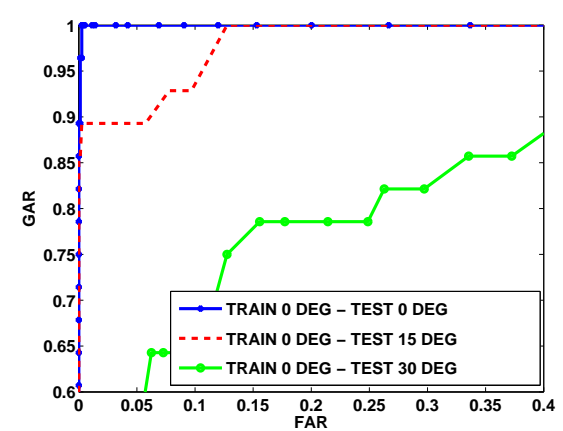

(a)

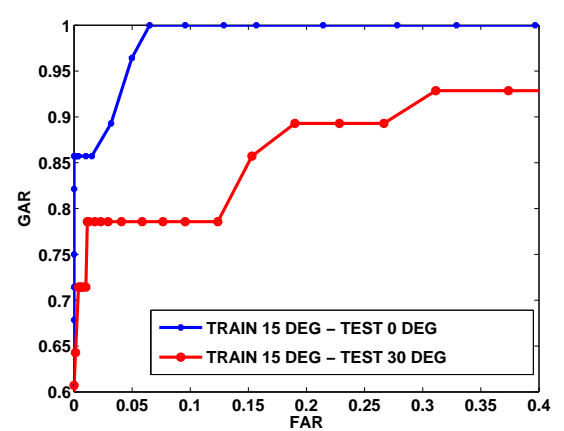

(b)

Figure 5.14: Performance of algorithms when using initial estimates for angles. (a). Train on 0 degree images and tested on others, and (b). Train on 15 degree images and tested on others

The results presented in Fig.5.15 were performed on the above mentioned database from WVU. It shows ROCs for different combinations of training and testing after compensating for two axes of rotation using the optimization technique (integrodifferential operator) to obtain the true estimate. Clearly, the performance has improved considerably over using just the initial estimate. However, the performance degrades as the difference in rotation between iris images increases significantly. One reason might be the interpolation stratergy adopted during projective transformation. Here, bicubic interpolation was used leading to highly smooth frontal view image for greater angles of rotation. This can be overcome with a better resampling technique.

\subsection{Other Studies on Encoding}

Apart from the above encoding results, a number of other experiments related to the system design have been conducted. These are performed on frontal view images, 


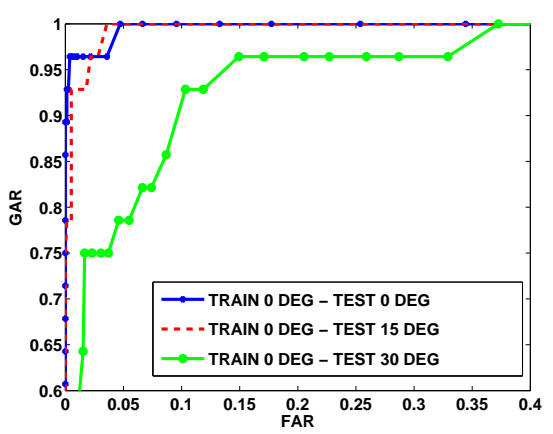

(a)

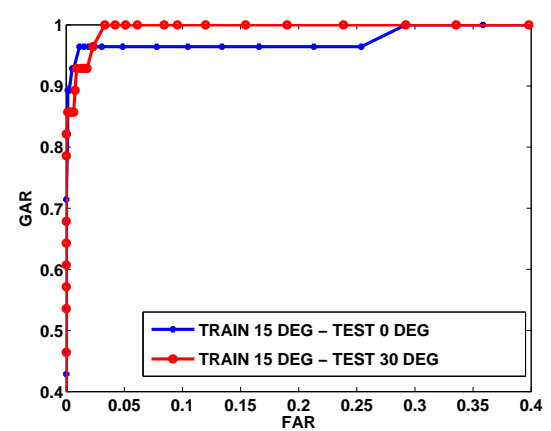

(b)

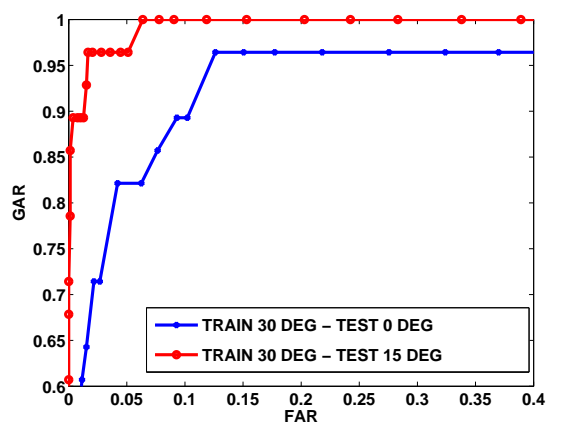

(c)

Figure 5.15: Performance of algorithms after compensating using the optimization technique (integro-differential operator). (a). Train on 0 degree images and tested on others (b). Train on 15 degree images and tested on others, and (c). Train on 30 degree images and tested on others 
predominantly using the CASIA dataset.

\subsubsection{How many Eigenvectors?}

In any application that uses PCA (or ICA), it is of great interest to analyze the number of eigenvectors required (or independent components in case of ICA). Hence, two experiments are carried out to investigate the number of eigen vectors extracted. All experiments, like others were conducted on normalized and enhanced iris images. The extracted eigenvalues are arranged in decreasing order of their absolute value. In Fig.5.12 the percentage of variance contributed by every eigenvalue extracted is plotted. In the first test, a reasonably large patch of 10x360 is selected for varying number of training classes. Though the dimensionality of the patch is 3600 , the number of essential dimensions for representation of the training images is dependent on the number of training classes as shown in the first panel. Out of a total of 3600 dimensions, the number of essential eigenvalues selected depends only on the number of training images used. Here, only the first 125 eigenvalues are shown as all the remaining eigenvalues were very close to zero. In another test, the number of training images was kept constant at 100 training images and the dimensionality of the patch is increased(to a very small extent) to see if any more meaningful eigenvalues can contribute to the variance of the high dimensional space. It can be seen from the second panel that though the dimensionality increases, the number of essential eigenvalues depends only on the number of training images used and not on the size of the patch(i.e.dimensionality). To re-emphasize, the results shown for varying dimensionality are only for small variation in dimensionality and not for any number of dimensions. Here again only the first 125 values are plotted. The experiment establishes that the number of essential principal components depends on the number of iris classes used for training.

\subsubsection{Eigen irises and Independent irises}

In Fig.5.16, some examples of the variations in the features extracted from PCA and ICA techniques are shown. It can be clearly seen that PCA components exhibit 


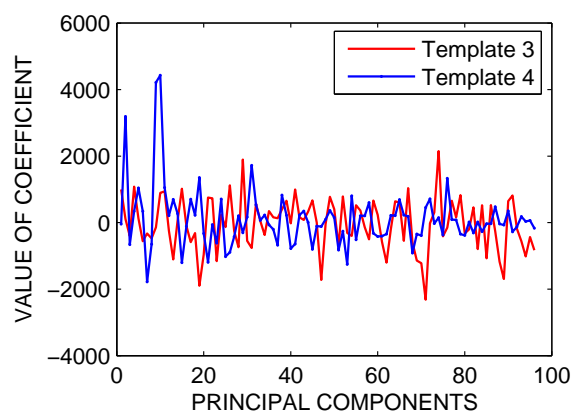

(a) PCA coefficients

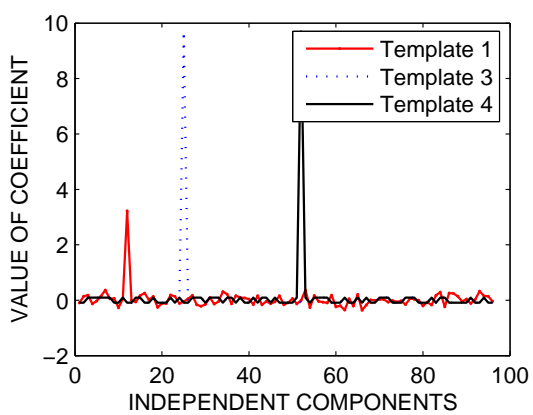

(b) ICA coefficients

Figure 5.16: Plot of image after projection into different trained subspaces

variation across different locations. The weighting coefficients from PCA(i.e.template values) give an idea about how close the incoming image resembles the trained basis function. Most of the basis functions seem to play a significant role in the final approximation to the input image in terms of the principal components. This is due to the fact that each of these bases contribute to a significant proportion of the total variance in comparison with the other selected bases. In sharp contrast, the projection coefficients from ICA tend to have a peaking value at some locations and very small values elsewhere. This suggests that the extracted independent components are highly similar to the training images at a very few locations and different than most ICs. Since the extracted components are independent and the training images similar to very few basis functions, it suggests that iris images themselves are statistically very different from each other(upto a few orders of statistics), thereby providing an implicit explanation of why iris structure offers such a good performance for recognition when we use statistics of lower orders. To say that each iris texture is totally different from the other irises may not always mean that the basic texture pattern is different (atleast not always). The underlying process in the generation of iris textures during birth is still the same which is the reason iris patterns are classified as iris patterns and can be distinguished from other texture patterns. Performance is a result of difference in spatial location of patterns which do not change dramatically over a life time.

As a result of the above experiments, one could propose that in continuum, each 


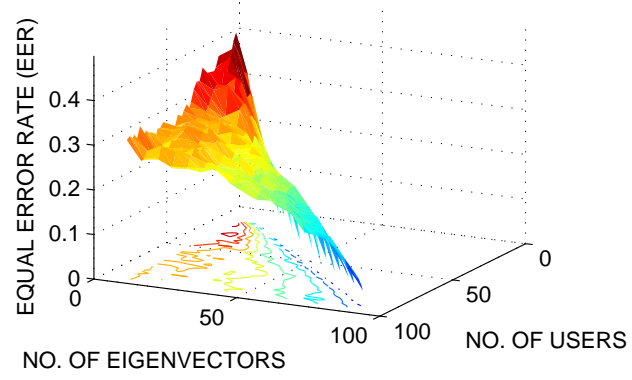

(a) PCA

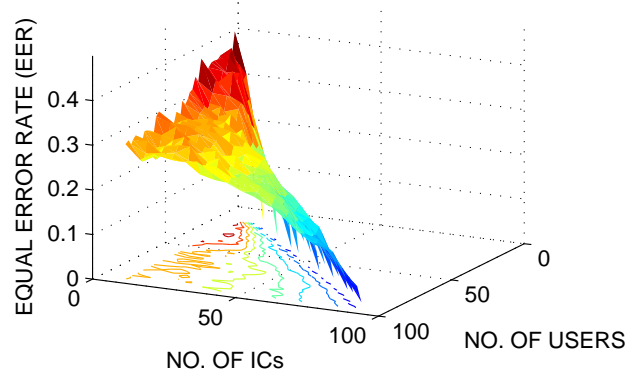

(b) ICA

Figure 5.17: Verification performance with varying number of essential components and number of users

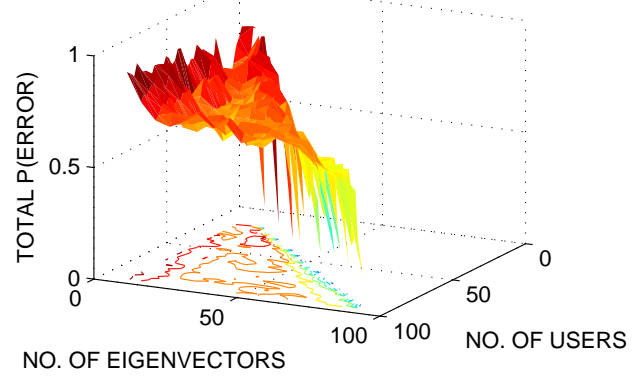

(a) PCA

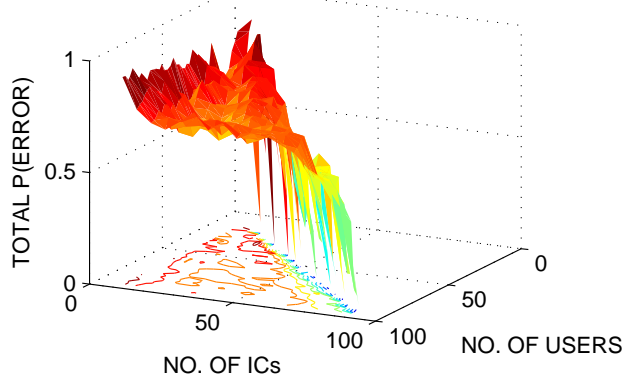

(b) ICA

Figure 5.18: Identification performance with varying number of essential components and number of users

new iris class adds a new orthonormal/independent axis and that in practice, the number of essential eigenirises is limited by iris image resolution and by preprocessing (ongoing research).

\subsubsection{Effect of Varying the Number of Essential Components}

To understand the effect of the number of essential components (essential eigenvalues in case of PCA and essential number of independent components in case of ICA), tests were conducted on the same database and the results are presented below in the form of a surface plot. 


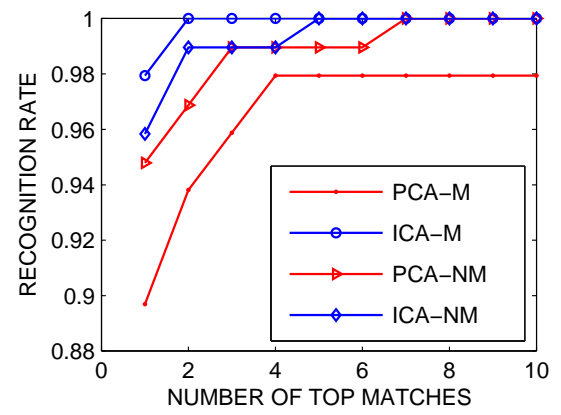

(a)

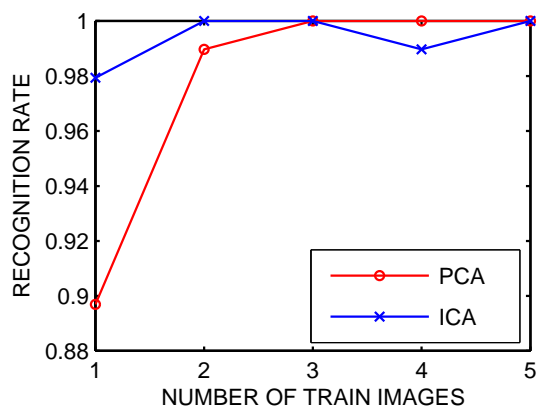

(b)

Figure 5.19: (a). Recognition performance with top N matches. M- with masking for occlusions, NM-without masking for occlusions. (b).Performance of proposed techniques as the number of training images in identification case. There is clearly an improvement in performance when using more training templates.

\section{Verification and Identification}

In Fig.5.17, verification results are presented. In Fig.5.18, identification results are presented. In the verification case, it can be seen that, for a given number of training images, as the number of Eigenvectors increases, the performance improves. Moreover, as the number of training images increases, the performance at lower number of Eigenvectors improves as well. But whenever (N-1) eigenvectors are used (where $\mathrm{N}$ is the number of training images, which is also the number of iris classes in this case) the performance reaches it best value. This emphasizes the importance of each eigenvector, even as the number of iris classes used for training increases which is due to the unique texture that each iris class possesses. It also reemphasizes the fact that the number of essential eigenvectors for $\mathrm{N}$ training images (where $\mathrm{N}$ is reasonably large, but not too large) is (N-1) as seen in the previous sections.

\subsubsection{Identification case with top $\mathrm{N}$ matches}

Identification performance can also be quantified based on "top N matches". Here, the probability of the true user's template being selected atleast once in the top $\mathrm{N}$ choices (that the system gives as output) is considered. For example, if there is a huge database of users to be compared against in an identification scenario, then the 
system comes up with a smaller list of $\mathrm{N}$ possible matches. Even if one of the templates from the possible matches correspond to the true user, we say that identification is achieved. This kind of matching is more useful and reasonable where there is a human inspector to make a final decision. The purpose is to reduce the workload of the human inspector. Fig.5.19 shows the graph for $\mathrm{N}$ versus identification rate, where $\mathrm{N}$ is the number of top matches selected. A total of 100 iris classes were considered.

\subsubsection{Relative Composition of Training and Testing Sets}

The relative composition of training and testing sets is one of importance to understand the performance of the system under different conditions that may exist in real world applications. Hence, the number of retained eigenvalues (here equal to the number of eigenvectors) is varied at different levels for each predetermined number of training images. It can be seen from Fig.5.19 that though the number of training images is lesser, as long as we retain enough number of eigenvectors we obtain good performance. It is also necessary to see the effect of including test images from imposters who are not enrolled in the system. In Fig.5.19, results corresponding to variation in relative composition of training and testing sets is provided.

In order to see this effect, we initially train on one image, test on another image from the same user. The number of training images per user is slowly increased while the same testing images are retained to verify how such training affects performance. Here, the overlap between genuine and imposter scores increases if we do not use the information that we may have more than one template of a given user. If the criterion that all templates present in the database for a given user should give a positive result is used(i.e. "AND" operation), then the performance would worsen as the number of templates used for training increases. This is because the incoming template faces a tougher challenge of being accepted by all the templates in the database. In case of identification results presented in Fig.5.19, even if one of the trained templates produces the lowest score, the person is considered identified(i.e. "OR" operation). It can be seen that as the number of training images increases, the performance also improves for both PCA and ICA. Hence, in verification mode, if a user is accepted even 


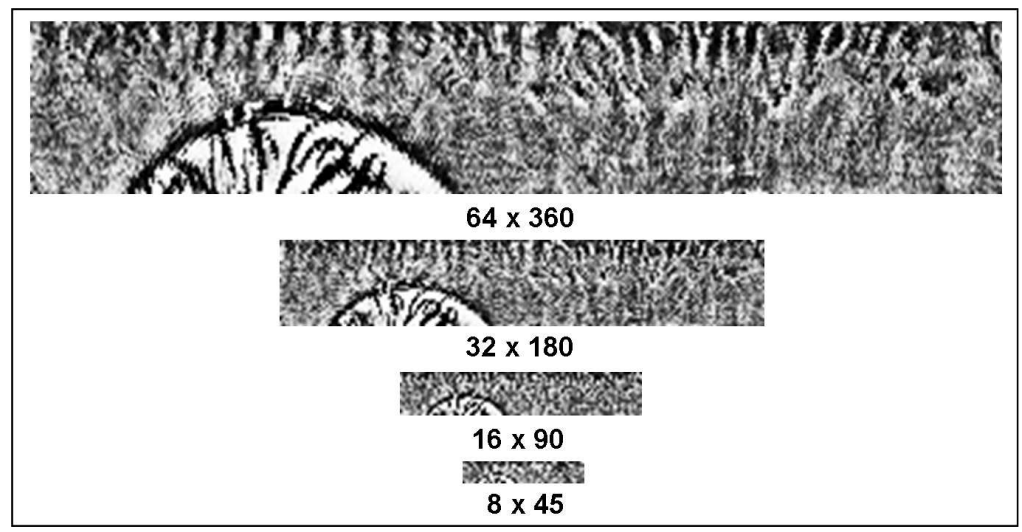

Figure 5.20: Downsampling of a normalized iris image

if one of the multiple matchscores fall below a certain threshold, then the performance would actually improve for the data at hand. Operations such as "AND", "OR" etc. are only simple techniques. With other smart ways of combining the different match scores, the performance would actually improve.

\subsubsection{Effect of Varying the Resolution}

In order to measure the effectiveness of the proposed algorithms based on PCA and ICA techniques, with respect to variations in resolution, we interpolate our iris images to a lower resolution grid. Two strategies of reducing image resolution were considered (i) down sampling and (ii) averaging. The first strategy is performed by selecting every second pixel in both the row and column directions. The second strategy is performed by averaging the original higher resolution image over non-overlapping blocks of size 2-by-2. Hence, in both the cases we have one point in the lower resolution grid for every four points in the next higher resolution grid. Fig.5.21 presents synthesized results. Empirical study of the effect of varying the resolution on the performance of the iris based identification system is an ongoing work at WVU. An example of downsampling a normalized iris image is presented in Fig.5.20. 


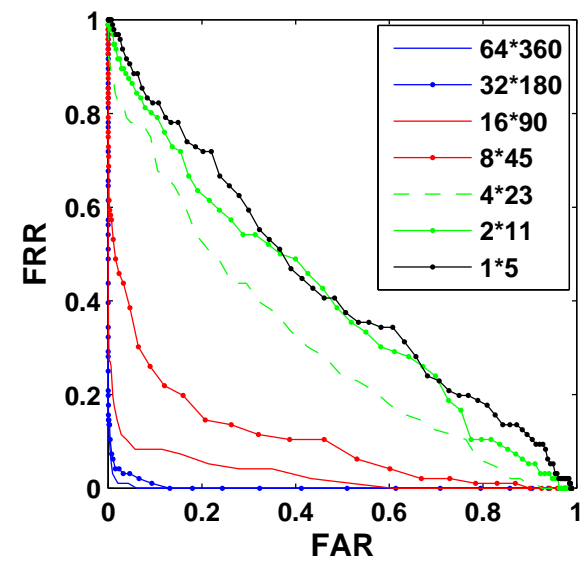

(a) PCA-Decimation

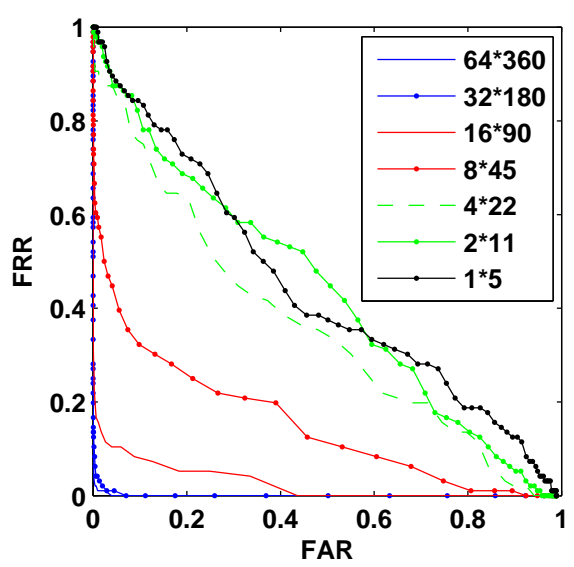

(c) ICA-Decimation

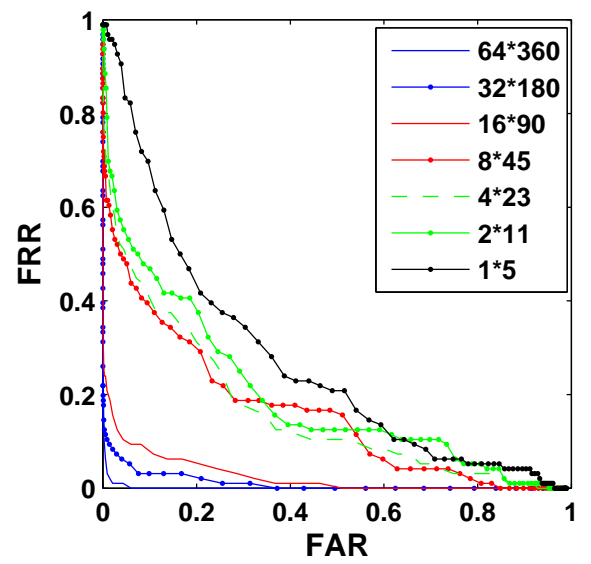

(b) PCA-Averaging

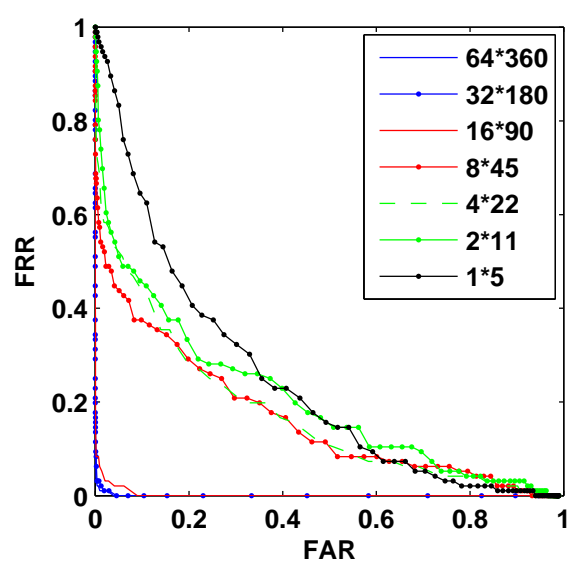

(d) ICA-Averaging

Figure 5.21: PCA/ICA results for various simulations on resolution 


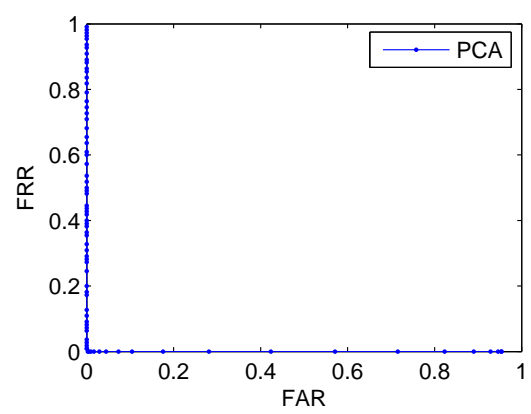

(a) PCA

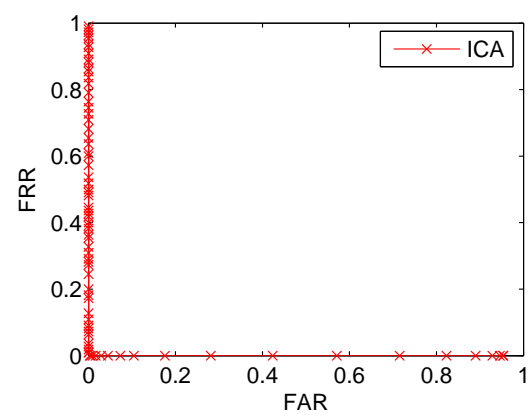

(b) ICA

Figure 5.22: Verification performance for WVU dataset with 22 iris classes and six images per user

\subsubsection{Other Databases and Distance Measures}

In order to verify verification performance on other databases, we performed experiments on 22 iris classes from a collection from our own labs. There are six templates per iris class. Experiment results as shown in Fig.5.22 confirm the good performance shown by both PCA and ICA methods. Distance measures that were discussed here were Euclidean and Hamming distance. Other distance measures can also be used, for example cosine distance, mahalanobis distance etc. These distances usually perform well but do not provide a great improvement over Euclidean distance.

\subsection{Effect of Specular Reflection on Performance}

The presence of specular reflection usually appears to be of little importance when considering performance of algorithms though presence of undetected specular reflection degrades performance as demonstrated below. Specular reflection affects performance by hindering with segmentation as well as by introducing spurious patterns on the iris. The results presented in Fig.5.23 were performed on a database with images containing specular reflection (22 iris classes with six images). It shows ROC curves for both

1. Images segmented without compensation for specular reflection, and 
2. Images segmented after detection and adapting to the presence of specular reflection.

As mentioned above, the reason for such a difference in performance is the difficulty in segmentation as a result of local maxima of criterion function (integro-differential operator) due to the presence of such specular reflection in iris images. Fig.5.23(a) shows Gabor filter technique with and without compensation. Fig.5.23(b) and Fig.5.23(c) shows results for PCA and ICA without compensation. Presence of specular reflection also reiterates the need for good segmentation in general. The effect is similar across different algorithms.

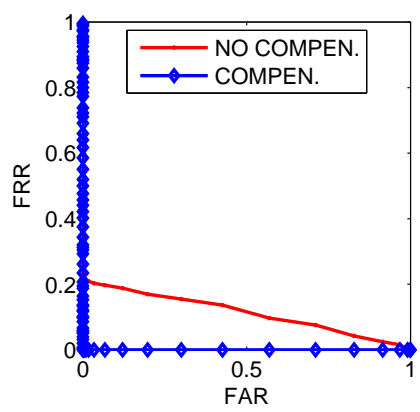

(a) Gabor filters

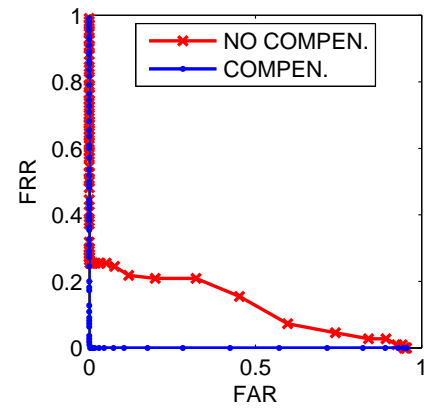

(b) PCA

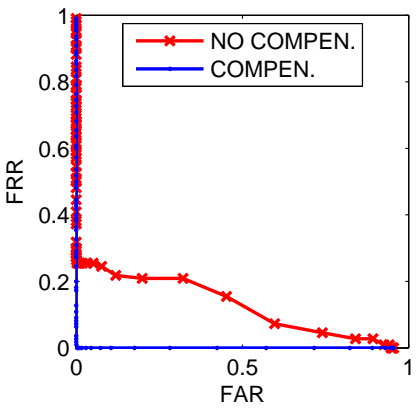

(c) ICA

Figure 5.23: Effect of presence of specular reflection on performance of algorithms 


\section{Chapter 6}

\section{Summary and Future Work}

\subsection{Summary}

Non-ideal iris data was defined and a new framework for non-ideal iris recognition has been proposed upon which future recognitions systems can be built. Using offangle iris data collected at WVU, it was shown that relative position of the acquisition device with respect to an individual's eye affects recognition performance significantly. Hence, an improved system was proposed to deal with off-angle images. The system operates in two stages. Firstly, the view of the individual is estimated using one of circular integro-differential operator, hamming distance and ellipse fitting techniques. Then the same image is transformed using projective geometry to bring the view of the off-axis iris image to frontal view. These images are then encoded as though they were frontal view images using global Principal Component Analysis and global Independent Component Analysis. The proposed technique was tested using off-axis data collected at WVU (after compensation) as well as other frontal view datasets using encoding methods developed for frontal view iris images. Based on the results,

it can be concluded that the proposed system improves recognition performance of off-axis iris data. It has also been shown that performance depends on various other factors apart from off-angle. 


\subsection{Future Work}

There are a lot of future directions for improving the system, especially its robustness. The model parameters used for recovering pose can be expanded to include the irissclera boundary and the eyelid boundary (which need to be estimated jointly with the transformation matrix). The position and size of specular reflections offer a way to estimate both the gaze of the individual w.r.t the camera as well as motion blur. But parameters will be dependent on various relative positions which need to be estimated. The number, positions and size of reflections will also play a role for camera specific estimations. The effect of the cornea and aqueous humor needs to be taken care of. Though a projective formulation has proved effective, the distortions due to the aqueous humour and the lens tend to have a significant impact on the final acquired image. This can be accounted for using modelling techniquesfor parameters specific to the human iris. The schematic shown in Fig.3.2 represents a generic nonideal iris recognition system. As mentioned earlier, effort should be made to reduce the number of blocks chosen out of this generic block diagram to build recognition systems without loss of performance. For example, it is possible to develop a system that would encode a given iris in such a way that most pre-processing steps such as compensation and normalization are not required, i.e. the encoding should be more invariant to variability in input. The quality assessment block needs to be developed to provide a reliable output that will be an integral part of future systems. If the need arises, compensation blocks can be developed to incorporate steps such as deblurring, super-resolution, fusing other available data etc. Robust segmentation is an essential step towards better recognition performance. Hence, segmentation algorithms need to be given greater importance for non-ideal data. 


\section{Bibliography}

[1] T. Mansfield, G. Kelly, D. Chandler, and J. Kane, "Biometric product testing final report," CESG/BWG Biometric Test Programme CESG contractX92A/4009309, Centre for Mathematics and Scientific Computing, National Physical Laboratory, Queens Road, March 2001.

[2] J. Daugman, "High confidence visual recognition of persons by a test of statistical independence," IEEE Transactions on Pattern Analysis and Machine Intelligence, vol. 15, no. 11, pp. 1148-1161, 1993.

[3] J. Daugman, "How iris recognition works," in ICIP02, 2002, pp. I: 33-36.

[4] J. Daugman, "The importance of being random: statistical principles of iris recognition," PR, vol. 36, no. 2, pp. 279-291, February 2003.

[5] J. Daugman, "Statistical richness of visual phase information: Update on recognizing persons by iris patterns," IJCV, vol. 45, no. 1, pp. 25-38, October 2001.

[6] Li Ma, T. Tan, Y. Wang, and D. Zhang, "Personal identification based on iris texture analysis," IEEE Transactions on Pattern Analysis and Machine Intelligence, vol. 25, pp. 1519-1533, 2003.

[7] R.P. Wildes, "Iris recognition: An emerging biometric technology," Proceedings of the IEEE, vol. 85, September 1997. 
[8] L. Masek, "Recognition of human iris patterns for biometric identification," B.S. Thesis, The University of Western Australia, http://www.csse.uwa.edu.au/pk/studentprojects/libor/, 2003.

[9] Junzhou Huang, Yunhong Wang, Tieniu Tan, and Jiali Cui, "A new iris segmentation method for recognition.," in $\operatorname{ICPR}$ (3), 2004, pp. 554-557.

[10] N. J. Ritter and J. R. Cooper, "Locating the iris: A first step to registration and identification," Proc. 9th IASTED International Conference on Signal and Image Processing, pp. 507-512, August 2003.

[11] Jiali Cui, Yunhong Wang, Tieniu Tan, Li Ma, and Zhenan Sun, "A fast and robust iris localization method based on texture segmentation," in Proc. SPIE, Anil K. Jain and Nalini K. Ratha, Eds., August 2004, vol. 5404 of Biometric Technology for Human Identification, pp. 401-408.

[12] W.W. Boles, "A security system based on human iris identification using wavelet transform," First International Conference on Knowledge-Based Intelligent Electronic Systems, pp. 533-541, May 1997, Adelaide, Australia.

[13] W.W. Boles and B. Boashash, "A human identification technique using images of the iris and wavelet transform," TSP, vol. 46, no. 4, pp. 1185-1188, April 1998.

[14] J.G. Daugman, "Two-dimensional spectral analysis of cortical receptive field profiles," Vision Research, vol. 20, pp. 847-856, 1980.

[15] J.G. Daugman, "Uncertainty relation for resolution in space, spatial frequency and orientation optimized by two-dimensional visual cortical filters," J. Opt. Soc. Am., vol. 2, no. 7, pp. 1160-1169, 1985.

[16] D.H. Kim and J.S. Ryoo, "Iris identification system and method of identifying a person through iris recognition," in US Patent, 1999.

[17] Y. Wang, T.N. Tan, and A.K. Jain, "Combining face and iris biometrics for identity verification," in AVBPA03, 2003, pp. 805-813. 
[18] C. Sanchez-Avila and R. Sanchez-Reillo, "Two different approaches for iris recognition using gabor filters and multiscale zero-crossing representation," $P R$, vol. 38, no. 2, pp. 231-240, February 2005.

[19] R. Sanchez-Reillo and C. Sanchez-Avila, "Iris recognition with low template size," in AVBPA01, 2001, p. 324.

[20] X. Ming, Z. Li, Z. Wang, and X. Zhu, "Multi-matching process based on wavelet transform for iris recognition," in WACV05, 2005, pp. I: 66-70.

[21] E. Krichen, M.A. Mellakh, S. Garcia-Salicetti, and B. Dorizzi, "Iris identification using wavelet packets," in ICPR04, 2004, pp. IV: 335-338.

[22] H. Sung, J. Lim, J.H. Park, and Y. Lee, "Iris recognition using collarette boundary localization," in ICPR04, 2004, pp. IV: 857-860.

[23] Z. Sun, Y. Wang, T.N. Tan, and J. Cui, "Robust direction estimation of gradient vector field for iris recognition," in ICPR04, 2004, pp. II: 783-786.

[24] J. de Mira and J. Mayer, "Identification of individuals through the morphological processing of the iris," in ICIP03, 2003, pp. I: 341-344.

[25] J. Jang, K. Kim, and Y. Lee, "Efficient algorithm of eye image check for robust iris recognition system," in CAIP03, 2003, pp. 301-308.

[26] B.V.K. Vijaya Kumar, C. Xie, and J. Thornton, "Iris verification using correlation filters," in AVBPA03, 2003, pp. 697-705.

[27] C.H. Park, J.J. Lee, S.K. Oh, Y.C. Song, D.H. Choi, and K.H. Park, "Iris feature extraction and matching based on multiscale and directional image representation," in ScaleSpace03, 2003, pp. 576-583.

[28] V. Vigneron, H. Maaref, and S. Lelandais, "Poor man vote with m-ary classifiers. application to iris recognition," in AVBPA03, 2003, pp. 647-657. 
[29] Ya-Ping Huang, Si-Wei Luo, and En-Yi Chen, "An efficient iris recognition system," in Proc. of the First International Conference on Machine Learning and Cybernetics, Beijing, Nov. 2002.

[30] Kwanghyuk Bae, Seung-In Noh, and Jaihie Kim, "Iris feature extraction using independent component analysis," in 4th International Conference Audio-and Video-Based Biometric Person Authentication, Guildford, UK, June 2003, pp. 838-844.

[31] Seung-In Noh, Kwanghuk Bae, Chulhan Lee, and Jaihie Kim, "Multiresolution independent component analysis for iris identification," in The 2002 International Technical Conference on Circuits/Systems, Computers and Communications, Phuket, Tailand, July 2002.

[32] E. Sung, X. Chen, J. Zhu, and J. Yang, "Towards non-cooperative iris recognition systems," in Proceedings of International Conference on Automation, Robotics, and Computer Vision (ICARCV'2002), 2002, vol. 2, pp. 990 - 995.

[33] C.N. Chun and R. Chung, "Iris recognition for iris tilted in depth," in CAIP03, 2003, pp. 530-539.

[34] Y. Du, B. Bonney, R. Ives, and D. M. Etter, "Analysis of partial iris recognition," in Proc. of the SPIE 2005 Symp. on Defense and Security, Orlando, FL., March Conf. 5779.

[35] A.S. Abhyankar, L.A. Hornak, and S. C. Schuckers, "Biorthogonal wavelet-based iris recognition," in Proc. of the SPIE 2005 Symp. on Defense and Security, Orlando, FL, March Conf. 5779.

[36] D.G. Lowe, "Three-dimensional object recognition from single two-dimensional images," Artif. Intell., vol. 31, no. 3, pp. 355-395, 1987.

[37] David G. Lowe, "Fitting parameterized three-dimensional models to images.," IEEE Trans. Pattern Anal. Mach. Intell., vol. 13, no. 5, pp. 441-450, 1991. 
[38] Masaru Ishii and Yoshio Mikami, "A 3-d sensor system for teaching robot paths and environments," Int. J. Rob. Res., vol. 6, no. 2, pp. 45-59, 1987.

[39] Araujo Helder, Rodrigo L. Carceroni, and Christopher M. Brown, "A fully projective formulation to improve the accuracy of lowe's pose estimation algorithm," Computer Vision and Image Understanding, vol. 70, no. 2, pp. 227-238, May 1998.

[40] Andrew Blake and Alan Yuille, Eds., Active vision, MIT Press, Cambridge, MA, USA, 1993.

[41] Alan L. Yuille, Peter W. Hallinan, and David S. Cohen, "Feature extraction from faces using deformable templates," Int. J. Comput. Vision, vol. 8, no. 2, pp. 99-111, 1992.

[42] Peter J. Burt and Edward H. Adelson, "The Laplacian pyramid as a compact image code," pp. 671-679, 1987.

[43] Igor Guskov, Wim Sweldens, and Peter Schröder, "Multiresolution signal processing for meshes," in SIGGRAPH '99: Proceedings of the 26th annual conference on Computer graphics and interactive techniques, New York, NY, USA, 1999, pp. 325-334, ACM Press/Addison-Wesley Publishing Co.

[44] T. Camus and R.P. Wildes, "Reliable and fast eye finding in close-up images," in Proc. of the IEEE International Conference on Pattern Recognition, Quebec City, Aug. 2002, pp. 389-394.

[45] G. Almkvist and B. Berndt, "Gauss, Landen, Ramanujan, the arithmetic geometric mean, ellipses, pi, and the ladies' diary," The American Mathematical Monthly, vol. 95, pp. 585-608, 1988.

[46] L. Sirovich and M. Kirby, "Low-dimensional procedure for the characterization of human faces," Journal of Optical Soc. Am, vol. 4, pp. 519-524, 1987. 
[47] M.A. Turk and A.P. Pentland, "Face recognition using eigenfaces," in Proceedings of the IEEE Conference on Computer Vision and Pattern Recognition, Hawaii, June 1992, p. 58690.

[48] M. Turk and Alex Pentland, "Eigenfaces for recognition," Journal of Cognitive Neuroscience, vol. 3, no. 1, pp. 71-86, 1991.

[49] A. Hyvärinen, J. Karhunen, and E. Oja, Independent Component Analysis, John Wiley and Sons, 2001.

[50] FastICA Matlab Package, Available online at http://www.cis.hut.fi/projects/ica/fastica.

[51] CASIA Iris Image Database (ver. 1.0), http://www.sinobiometrics.com/casiairis.htm.

[52] Vivekanand Dorairaj, Yash N. Shah, and Natalia A. Schmid, "Exploring and developing processing techniques for iris recognition system," in Biometric Consortium Conference. 2004, http://www.wvu.edu/ bknc/2004 Abstracts/Biometric Symposium Abstracts public2 htm.

[53] Bruce A. Draper, Kyungim Baek, Marian Stewart Bartlett, and J. Ross Beveridge, "Recognizing faces with PCA and ICA," in CVIU, June 2003, pp. $115-137$.

[54] Juha Koikkalainen and Jyrki Lotjonen, "Image segmentation with the combination of the PCA and ICA based modes of shape variation," ISBI, pp. 149-152, 2004.

[55] P.D. Imesch, I.H.L. Wallow, and D.M. Albert, "The color of the human eye: A review of morphologic correlates and of some conditions that affect iridial pigmentation," Survey of Opthalmology, vol. 41, no. 2, pp. S117-S123, February 1997.

[56] John Forrester, A.D. Dick, P. McMenamin, and W. Lee, The Eye: Basic Sciences in Practice,2nd ed., Saunders, October 2001. 
[57] M. Kirby and L. Sirovich, "Application of the karhunen-loeve procedure for the characterization of human face," IEEE, vol. 12, pp. 103-108, 1990.

[58] H. Le Borgne, A. Guerin-Dugue, and A. Antoniadis, "Representation of images for classification with independent features," PRL, vol. 25, no. 2, pp. 141-154, January 2004.

[59] Aapo Hyvärinen, "Independent component analysis in the presence of gaussian noise by maximizing joint likelihood," Neurocomputing, vol. 22, pp. 49-67, 1998.

[60] Jean-Franois Cardoso, "Infomax and maximum likelihood for source separation," IEEE Letters on Signal Processing, vol. 4, no. 4, pp. 112-114, 1997.

[61] Antony J. Bell and Terrence J. Sejnowski, "An information maximisation approach to blind separation and blind deconvolution," Neural Computation, vol. 7, no. 6, pp. 1129-1159, 1995.

[62] A.J. Bell and T.J. Sejnowski, "The 'independent components' of natural scenes are edge filters," Vision Research, , no. 37, pp. 3327-3338, 1997.

[63] K. Sengupta, P. Burman, and R. Sharma, "A non-parametric approach for independent component analysis using kernel density estimation," in CVPR04, 2004, pp. II: 667-672.

[64] C. Fang and X. Ding, "Improvement of ica based probability density estimation for pattern recognition," in ICPR04, 2004, pp. III: 466-469.

[65] A.J. Ferreira and M.A.T. Figueiredo, "Class-adapted image compression using independent component analysis," in ICIP03, 2003, pp. I: 625-628.

[66] M. Bressan, D. Guillamet, and J. Vitria, "Using an ica representation of high dimensional data for object recognition and classification," in CVPR01, 2001, pp. I:1004-1009.

[67] J. Fortuna, D. Schuurman, and D. Capson, "A comparison of pca and ica for object recognition under varying illumination," in ICPR02, 2002, pp. III: 11-15. 
[68] Aapo Hyvärinen, Patrik O. Hoyer, and Mika Inki, "Topographic ica as a model of natural image statistics," in BMVC '00: Proceedings of the First IEEE International Workshop on Biologically Motivated Computer Vision. 2000, pp. 535-544, Springer-Verlag.

[69] Shun Ichi Amari, Aapo Hyvärinen, Soo Young Lee, Te Won Lee, and V. David Sánchez A., "Blind signal separation and independent component analysis.," Neurocomputing, vol. 49, no. 1-4, pp. 1-5, 2002.

[70] Jagath C. Rajapakse, "Adaptive blind signal and image processing: learning algorithms and applications: A. cichocki, s. amari, wiley, new york, 2002, 586pp., isbn 047160791 6.," Neurocomputing, vol. 49, no. 1-4, pp. 439-443, 2002.

[71] Erkki Oja and Juha Karhunen, "Nonlinear PCA: Algorithms and applications," Tech. Rep. A18, Helsinki University of Technology Laboratory of Computer and Information Sciences, Rakentajanaukio 2 C, SF-02150 Espoo, Finland, Sept. 1993.

[72] Thomas M. Cover and Joy A. Thomas, Elements of Information Theory, John Wiley \& sons, 1991.

[73] S. Makeig, Anthony J. Bell, Tzyy-Ping Jung, and Terrence J. Sejnowski, "Independent component analysis of electroencephalographic data," Advances in Neural Information Processing Systems, vol. 8, pp. 145-151, 1996.

[74] Nicolas Brunel and Jean-Pierre Nadal, "Mutual information, fisher information and population coding," Neural Computation, vol. 10, no. 7, pp. 1731-1757, 1998. 